\title{
Affine transformations of a Leonard pair
}

\author{
Kazumasa Nomura and Paul Terwilliger
}

\begin{abstract}
Let $\mathbb{K}$ denote a field and let $V$ denote a vector space over $\mathbb{K}$ with finite positive dimension. We consider an ordered pair of linear transformations $A: V \rightarrow V$ and $A^{*}: V \rightarrow V$ that satisfy (i) and (ii) below:

(i) There exists a basis for $V$ with respect to which the matrix representing $A$ is irreducible tridiagonal and the matrix representing $A^{*}$ is diagonal.

(ii) There exists a basis for $V$ with respect to which the matrix representing $A^{*}$ is irreducible tridiagonal and the matrix representing $A$ is diagonal.

We call such a pair a Leonard pair on $V$. Let $\xi, \zeta, \xi^{*}, \zeta^{*}$ denote scalars in $\mathbb{K}$ with $\xi, \xi^{*}$ nonzero, and note that $\xi A+\zeta I, \xi^{*} A^{*}+\zeta^{*} I$ is a Leonard pair on $V$. We give necessary and sufficient conditions for this Leonard pair to be isomorphic to $A, A^{*}$. We also give necessary and sufficient conditions for this Leonard pair to be isomorphic to the Leonard pair $A^{*}, A$.
\end{abstract}

\section{Leonard pairs}

We begin by recalling the notion of a Leonard pair. We will use the following terms. A square matrix $X$ is said to be tridiagonal whenever each nonzero entry lies on either the diagonal, the subdiagonal, or the superdiagonal. Assume $X$ is tridiagonal. Then $X$ is said to be irreducible whenever each entry on the subdiagonal is nonzero and each entry on the superdiagonal is nonzero. We now define a Leonard pair. For the rest of this paper $\mathbb{K}$ will denote a field.

Definition 1.1 29] Let $V$ denote a vector space over $\mathbb{K}$ with finite positive dimension. By a Leonard pair on $V$ we mean an ordered pair $A, A^{*}$ where $A: V \rightarrow V$ and $A^{*}: V \rightarrow V$ are linear transformations that satisfy (i) and (ii) below:

(i) There exists a basis for $V$ with respect to which the matrix representing $A$ is irreducible tridiagonal and the matrix representing $A^{*}$ is diagonal.

(ii) There exists a basis for $V$ with respect to which the matrix representing $A^{*}$ is irreducible tridiagonal and the matrix representing $A$ is diagonal.

Note 1.2 It is a common notational convention to use $A^{*}$ to represent the conjugatetranspose of $A$. We are not using this convention. In a Leonard pair $A, A^{*}$ the linear transformations $A$ and $A^{*}$ are arbitrary subject to (i) and (ii) above.

We refer the reader to [5], [15], [18, [19, 20], 21], 22], 23], 24], 27], 28, [29], 31, [32], 33], 34], 35], 36], 37], 38, [40], 44], 42] for background on Leonard pairs. We especially recommend the survey [38]. See [1], [2], [3], 4], [6], [7], [8], [9], [10, 11], [12], [13], [14], [16], 17], 25], 26], 30], 39], [43] for related topics. 
In this paper we consider the following situation. Let $V$ denote a vector space over $\mathbb{K}$ with finite positive dimension and let $A, A^{*}$ denote a Leonard pair on $V$. Let $\xi, \zeta, \xi^{*}, \zeta^{*}$ denote scalars in $\mathbb{K}$ with $\xi, \xi^{*}$ nonzero, and note that $\xi A+\zeta I, \xi^{*} A^{*}+\zeta^{*} I$ is a Leonard pair on $V$. We give necessary and sufficient conditions for this Leonard pair to be isomorphic to $A, A^{*}$. We also give necessary and sufficient conditions for this Leonard pair to be isomorphic to the Leonard pair $A^{*}, A$.

\section{Leonard systems}

When working with a Leonard pair, it is convenient to consider a closely related object called a Leonard system. To prepare for our definition of a Leonard system, we recall a few concepts from linear algebra. Let $d$ denote a nonnegative integer and let $\operatorname{Mat}_{d+1}(\mathbb{K})$ denote the $\mathbb{K}$-algebra consisting of all $d+1$ by $d+1$ matrices that have entries in $\mathbb{K}$. We index the rows and columns by $0,1, \ldots, d$. We let $\mathbb{K}^{d+1}$ denote the $\mathbb{K}$-vector space of all $d+1$ by 1 matrices that have entries in $\mathbb{K}$. We index the rows by $0,1, \ldots, d$. We view $\mathbb{K}^{d+1}$ as a left module for $\operatorname{Mat}_{d+1}(\mathbb{K})$. We observe this module is irreducible. For the rest of this paper, let $\mathcal{A}$ denote a $\mathbb{K}$-algebra isomorphic to $\operatorname{Mat}_{d+1}(\mathbb{K})$ and let $V$ denote an irreducible left $\mathcal{A}$-module. We remark that $V$ is unique up to isomorphism of $\mathcal{A}$-modules, and that $V$ has dimension $d+1$. Let $\left\{v_{i}\right\}_{i=0}^{d}$ denote a basis for $V$. For $X \in \mathcal{A}$ and $Y \in \operatorname{Mat}_{d+1}(\mathbb{K})$, we say $Y$ represents $X$ with respect to $\left\{v_{i}\right\}_{i=0}^{d}$ whenever $X v_{j}=\sum_{i=0}^{d} Y_{i j} v_{i}$ for $0 \leq j \leq d$. For $A \in \mathcal{A}$ we say $A$ is multiplicity-free whenever it has $d+1$ mutually distinct eigenvalues in $\mathbb{K}$. Assume $A$ is multiplicity-free. Let $\left\{\theta_{i}\right\}_{i=0}^{d}$ denote an ordering of the eigenvalues of $A$, and for $0 \leq i \leq d$ put

$$
E_{i}=\prod_{\substack{0 \leq j \leq d \\ j \neq i}} \frac{A-\theta_{j} I}{\theta_{i}-\theta_{j}}
$$

where $I$ denotes the identity of $\mathcal{A}$. We observe (i) $A E_{i}=\theta_{i} E_{i}(0 \leq i \leq d)$; (ii) $E_{i} E_{j}=\delta_{i, j} E_{i}$ $(0 \leq i, j \leq d)$; (iii) $\sum_{i=0}^{d} E_{i}=I$; (iv) $A=\sum_{i=0}^{d} \theta_{i} E_{i}$. Let $\mathcal{D}$ denote the subalgebra of $\mathcal{A}$ generated by $A$. Using (i)-(iv) we find the sequence $\left\{E_{i}\right\}_{i=0}^{d}$ is a basis for the $\mathbb{K}$-vector space $\mathcal{D}$. We call $E_{i}$ the primitive idempotent of $A$ associated with $\theta_{i}$. It is helpful to think of these primitive idempotents as follows. Observe

$$
V=E_{0} V+E_{1} V+\cdots+E_{d} V \quad \text { (direct sum). }
$$

For $0 \leq i \leq d, E_{i} V$ is the (one dimensional) eigenspace of $A$ in $V$ associated with the eigenvalue $\theta_{i}$, and $E_{i}$ acts on $V$ as the projection onto this eigenspace.

By a Leonard pair in $\mathcal{A}$ we mean an ordered pair of elements taken from $\mathcal{A}$ that act on $V$ as a Leonard pair in the sense of Definition 1.1. We call $\mathcal{A}$ the ambient algebra of the pair and say the pair is over $\mathbb{K}$. We now define a Leonard system. 
Definition 2.1 [29] By a Leonard system in $\mathcal{A}$ we mean a sequence

$$
\Phi=\left(A ;\left\{E_{i}\right\}_{i=0}^{d} ; A^{*} ;\left\{E_{i}^{*}\right\}_{i=0}^{d}\right)
$$

that satisfies (i)-(v) below.

(i) Each of $A, A^{*}$ is a multiplicity-free element in $\mathcal{A}$.

(ii) $\left\{E_{i}\right\}_{i=0}^{d}$ is an ordering of the primitive idempotents of $A$.

(iii) $\left\{E_{i}^{*}\right\}_{i=0}^{d}$ is an ordering of the primitive idempotents of $A^{*}$.

(iv) For $0 \leq i, j \leq d$,

$$
E_{i} A^{*} E_{j}= \begin{cases}0 & \text { if }|i-j|>1 \\ \neq 0 & \text { if }|i-j|=1\end{cases}
$$

(v) For $0 \leq i, j \leq d$,

$$
E_{i}^{*} A E_{j}^{*}= \begin{cases}0 & \text { if }|i-j|>1, \\ \neq 0 & \text { if }|i-j|=1 .\end{cases}
$$

We refer to $d$ as the diameter of $\Phi$ and say $\Phi$ is over $\mathbb{K}$. We call $\mathcal{A}$ the ambient algebra of $\Phi$.

Leonard systems are related to Leonard pairs as follows. Let $\left(A ;\left\{E_{i}\right\}_{i=0}^{d} ; A^{*} ;\left\{E_{i}^{*}\right\}_{i=0}^{d}\right)$ denote a Leonard system in $\mathcal{A}$. Then $A, A^{*}$ is a Leonard pair in $\mathcal{A}$ [37, Section 3]. Conversely, suppose $A, A^{*}$ is a Leonard pair in $\mathcal{A}$. Then each of $A, A^{*}$ is multiplicity-free [29, Lemma 1.3]. Moreover there exists an ordering $\left\{E_{i}\right\}_{i=0}^{d}$ of the primitive idempotents of $A$, and there exists an ordering $\left\{E_{i}^{*}\right\}_{i=0}^{d}$ of the primitive idempotents of $A^{*}$, such that $\left(A ;\left\{E_{i}\right\}_{i=0}^{d} ; A^{*} ;\left\{E_{i}^{*}\right\}_{i=0}^{d}\right)$ is a Leonard system in $\mathcal{A}$ [37, Lemma 3.3]. We say this Leonard system is associated with the Leonard pair $A, A^{*}$.

We recall the notion of isomorphism for Leonard pairs and Leonard systems.

Definition 2.2 Let $A, A^{*}$ and $B, B^{*}$ denote Leonard pairs over $\mathbb{K}$. By an isomorphism of Leonard pairs from $A, A^{*}$ to $B, B^{*}$ we mean an isomorphism of $\mathbb{K}$-algebras from the ambient algebra of $A, A^{*}$ to the ambient algebra $B, B^{*}$ that sends $A$ to $B$ and $A^{*}$ to $B^{*}$. The Leonard pairs $A, A^{*}$ and $B, B^{*}$ are said to be isomorphic whenever there exists an isomorphism of Leonard pairs from $A, A^{*}$ to $B, B^{*}$.

Let $\Phi$ denote the Leonard system from Definition 2.1 and let $\sigma: \mathcal{A} \rightarrow \mathcal{A}^{\prime}$ denote an isomorphism of $\mathbb{K}$-algebras. We write $\Phi^{\sigma}:=\left(A^{\sigma} ;\left\{E_{i}^{\sigma}\right\}_{i=0}^{d} ; A^{* \sigma} ;\left\{E_{i}^{* \sigma}\right\}_{i=0}^{d}\right)$ and observe $\Phi^{\sigma}$ is a Leonard system in $\mathcal{A}^{\prime}$.

Definition 2.3 Let $\Phi$ and $\Phi^{\prime}$ denote Leonard systems over $\mathbb{K}$. By an isomorphism of Leonard systems from $\Phi$ to $\Phi^{\prime}$ we mean an isomorphism of $\mathbb{K}$-algebras $\sigma$ from the ambient algebra of $\Phi$ to the ambient algebra of $\Phi^{\prime}$ such that $\Phi^{\sigma}=\Phi^{\prime}$. The Leonard systems $\Phi$ and $\Phi^{\prime}$ are said to be isomorphic whenever there exists an isomorphism of Leonard systems from $\Phi$ to $\Phi^{\prime}$. 


\section{The $D_{4}$ action}

Let $\Phi=\left(A ;\left\{E_{i}\right\}_{i=0}^{d} ; A^{*} ;\left\{E_{i}^{*}\right\}_{i=0}^{d}\right)$ denote a Leonard system in $\mathcal{A}$. Then each of the following is a Leonard system in $\mathcal{A}$ :

$$
\begin{aligned}
& \Phi^{*}:=\left(A^{*} ;\left\{E_{i}^{*}\right\}_{i=0}^{d} ; A ;\left\{E_{i}\right\}_{i=0}^{d}\right), \\
& \Phi^{\downarrow}:=\left(A ;\left\{E_{i}\right\}_{i=0}^{d} ; A^{*} ;\left\{E_{d-i}^{*}\right\}_{i=0}^{d}\right), \\
& \Phi^{\Downarrow}:=\left(A ;\left\{E_{d-i}\right\}_{i=0}^{d} ; A^{*} ;\left\{E_{i}^{*}\right\}_{i=0}^{d}\right) .
\end{aligned}
$$

Viewing $*, \downarrow, \Downarrow$ as permutations on the set of all the Leonard systems,

$$
\begin{gathered}
*^{2}=\downarrow^{2}=\Downarrow^{2}=1, \\
\Downarrow *=* \downarrow, \quad \downarrow *=* \Downarrow, \quad \downarrow \Downarrow=\Downarrow \downarrow .
\end{gathered}
$$

The group generated by symbols $*, \downarrow, \Downarrow$ subject to the relations (4), (5) is the dihedral group $D_{4}$. We recall $D_{4}$ is the group of symmetries of a square, and has 8 elements. Apparently $*, \downarrow, \Downarrow$ induce an action of $D_{4}$ on the set of all Leonard systems. Two Leonard systems will be called relatives whenever they are in the same orbit of this $D_{4}$ action. The relatives of $\Phi$ are as follows:

\begin{tabular}{c|c} 
name & relative \\
\hline$\Phi$ & $\left(A ;\left\{E_{i}\right\}_{i=0}^{d} ; A^{*} ;\left\{E_{i}^{*}\right\}_{i=0}^{d}\right)$ \\
$\Phi^{\downarrow}$ & $\left(A ;\left\{E_{i}\right\}_{i=0}^{d} ; A^{*} ;\left\{E_{d-i}^{*}\right\}_{i=0}^{d}\right)$ \\
$\Phi^{\Downarrow}$ & $\left(A ;\left\{E_{d-i}\right\}_{i=0}^{d} ; A^{*} ;\left\{E_{i}^{*}\right\}_{i=0}^{d}\right)$ \\
$\Phi^{\downarrow \Downarrow}$ & $\left(A ;\left\{E_{d-i}\right\}_{i=0}^{d} ; A^{*} ;\left\{E_{d-i}^{*}\right\}_{i=0}^{d}\right)$ \\
$\Phi^{*}$ & $\left(A^{*} ;\left\{E_{i}^{*}\right\}_{i=0}^{d} ; A ;\left\{E_{i}\right\}_{i=0}^{d}\right)$ \\
$\Phi^{\downarrow *}$ & $\left(A^{*} ;\left\{E_{d-i}^{*}\right\}_{i=0}^{d} ; A ;\left\{E_{i}\right\}_{i=0}^{d}\right)$ \\
$\Phi^{\Downarrow *}$ & $\left(A^{*} ;\left\{E_{i}^{*}\right\}_{i=0}^{d} ; A ;\left\{E_{d-i}\right\}_{i=0}^{d}\right)$ \\
$\Phi^{\downarrow \Downarrow *}$ & $\left(A^{*} ;\left\{E_{d-i}^{*}\right\}_{i=0}^{d} ; A ;\left\{E_{d-i}\right\}_{i=0}^{d}\right)$
\end{tabular}

\section{The parameter array}

In this section we recall the parameter array of a Leonard system.

Definition 4.1 Let $\Phi=\left(A ;\left\{E_{i}\right\}_{i=0}^{d} ; A^{*} ;\left\{E_{i}^{*}\right\}_{i=0}^{d}\right)$ denote a Leonard system over $\mathbb{K}$. For $0 \leq i \leq d$ we let $\theta_{i}$ (resp. $\theta_{i}^{*}$ ) denote the eigenvalue of $A$ (resp. $A^{*}$ ) associated with $E_{i}$ (resp. $E_{i}^{*}$ ). We refer to $\left\{\theta_{i}\right\}_{i=0}^{d}$ (resp. $\left\{\theta_{i}^{*}\right\}_{i=0}^{d}$ ) as the eigenvalue sequence (resp. dual eigenvalue sequence) of $\Phi$. We observe $\left\{\theta_{i}\right\}_{i=0}^{d}$ (resp. $\left.\left\{\theta_{i}^{*}\right\}_{i=0}^{d}\right)$ are mutually distinct and contained in $\mathbb{K}$. 
Definition 4.2 [19, Theorem 4.6] Let $\Phi=\left(A ;\left\{E_{i}\right\}_{i=0}^{d} ; A^{*} ;\left\{E_{i}^{*}\right\}_{i=0}^{d}\right)$ denote a Leonard system with eigenvalue sequence $\left\{\theta_{i}\right\}_{i=0}^{d}$ and dual eigenvalue sequence $\left\{\theta_{i}^{*}\right\}_{i=0}^{d}$. For $1 \leq$ $i \leq d$ we define

$$
\begin{aligned}
\varphi_{i} & :=\left(\theta_{0}^{*}-\theta_{i}^{*}\right) \frac{\operatorname{tr}\left(E_{0}^{*} \prod_{h=0}^{i-1}\left(A-\theta_{h} I\right)\right)}{\operatorname{tr}\left(E_{0}^{*} \prod_{h=0}^{i-2}\left(A-\theta_{h} I\right)\right)}, \\
\phi_{i} & :=\left(\theta_{0}^{*}-\theta_{i}^{*}\right) \frac{\operatorname{tr}\left(E_{0}^{*} \prod_{h=0}^{i-1}\left(A-\theta_{d-h} I\right)\right)}{\operatorname{tr}\left(E_{0}^{*} \prod_{h=0}^{i-2}\left(A-\theta_{d-h} I\right)\right)},
\end{aligned}
$$

where tr means trace. In (6), (7) the denominators are nonzero by [19, Corollary 4.5]. The sequence $\left\{\varphi_{i}\right\}_{i=1}^{d}$ (resp. $\left\{\phi_{i}\right\}_{i=1}^{d}$ ) is called the first split sequence (resp. second split sequence) of $\Phi$.

Definition 4.3 Let $\Phi=\left(A ;\left\{E_{i}\right\}_{i=0}^{d} ; A^{*} ;\left\{E_{i}^{*}\right\}_{i=0}^{d}\right)$ denote a Leonard system over $\mathbb{K}$. By the parameter array of $\Phi$ we mean the sequence $\left(\left\{\theta_{i}\right\}_{i=0}^{d} ;\left\{\theta_{i}^{*}\right\}_{i=0}^{d} ;\left\{\varphi_{i}\right\}_{i=1}^{d} ;\left\{\phi_{i}\right\}_{i=1}^{d}\right)$, where the $\theta_{i}, \theta_{i}^{*}$ are from Definition 4.1 and the $\varphi_{i}, \phi_{i}$ are from Definition 4.2 .

Theorem 4.4 [29, Theorem 1.9] Let d denote a nonnegative integer and let

$$
\left(\left\{\theta_{i}\right\}_{i=0}^{d} ;\left\{\theta_{i}^{*}\right\}_{i=0}^{d} ;\left\{\varphi_{i}\right\}_{i=1}^{d} ;\left\{\phi_{i}\right\}_{i=1}^{d}\right)
$$

denote a sequence of scalars taken from $\mathbb{K}$. Then there exists a Leonard system $\Phi$ over $\mathbb{K}$ with parameter array (8) if and only if (PA1)-(PA5) hold below.

(PA1) $\varphi_{i} \neq 0, \phi_{i} \neq 0(1 \leq i \leq d)$.

(PA2) $\theta_{i} \neq \theta_{j}, \theta_{i}^{*} \neq \theta_{j}^{*}$ if $i \neq j(0 \leq i, j \leq d)$.

(PA3) For $1 \leq i \leq d$,

$$
\varphi_{i}=\phi_{1} \sum_{h=0}^{i-1} \frac{\theta_{h}-\theta_{d-h}}{\theta_{0}-\theta_{d}}+\left(\theta_{i}^{*}-\theta_{0}^{*}\right)\left(\theta_{i-1}-\theta_{d}\right) .
$$

(PA4) For $1 \leq i \leq d$,

$$
\phi_{i}=\varphi_{1} \sum_{h=0}^{i-1} \frac{\theta_{h}-\theta_{d-h}}{\theta_{0}-\theta_{d}}+\left(\theta_{i}^{*}-\theta_{0}^{*}\right)\left(\theta_{d-i+1}-\theta_{0}\right) .
$$

(PA5) The expressions

$$
\frac{\theta_{i-2}-\theta_{i+1}}{\theta_{i-1}-\theta_{i}}, \quad \frac{\theta_{i-2}^{*}-\theta_{i+1}^{*}}{\theta_{i-1}^{*}-\theta_{i}^{*}}
$$

are equal and independent of $i$ for $2 \leq i \leq d-1$.

Suppose (PA1)-(PA5) hold. Then $\Phi$ is unique up to isomorphism of Leonard systems.

The $D_{4}$ action affects the parameter array as follows. 
Lemma 4.5 [29, Theorem 1.11] Let $\Phi=\left(A ;\left\{E_{i}\right\}_{i=0}^{d} ; A^{*} ;\left\{E_{i}^{*}\right\}_{i=0}^{d}\right)$ denote a Leonard system with parameter array $\left(\left\{\theta_{i}\right\}_{i=0}^{d} ;\left\{\theta_{i}^{*}\right\}_{i=0}^{d} ;\left\{\varphi_{i}\right\}_{i=1}^{d} ;\left\{\phi_{i}\right\}_{i=1}^{d}\right)$. For each relative of $\Phi$ the parameter array is given below.

\begin{tabular}{c|c} 
relative & parameter array \\
\hline$\Phi$ & $\left(\left\{\theta_{i}\right\}_{i=0}^{d} ;\left\{\theta_{i}^{*}\right\}_{i=0}^{d} ;\left\{\varphi_{i}\right\}_{i=1}^{d} ;\left\{\phi_{i}\right\}_{i=1}^{d}\right)$ \\
$\Phi^{\downarrow}$ & $\left(\left\{\theta_{i}\right\}_{i=0}^{d} ;\left\{\theta_{d-i}^{*}\right\}_{i=0}^{d} ;\left\{\phi_{d-i+1}\right\}_{i=1}^{d} ;\left\{\varphi_{d-i+1}\right\}_{i=1}^{d}\right)$ \\
$\Phi^{\Downarrow}$ & $\left(\left\{\theta_{d-i}\right\}_{i=0}^{d} ;\left\{\theta_{i}^{*}\right\}_{i=0}^{d} ;\left\{\phi_{i}\right\}_{i=1}^{d} ;\left\{\varphi_{i}\right\}_{i=1}^{d}\right)$ \\
$\Phi^{\downarrow \Downarrow}$ & $\left(\left\{\theta_{d-i}\right\}_{i=0}^{d} ;\left\{\theta_{d-i}^{*}\right\}_{i=0}^{d} ;\left\{\varphi_{d-i+1}\right\}_{i=1}^{d} ;\left\{\phi_{d-i+1}\right\}_{i=1}^{d}\right)$ \\
$\Phi^{*}$ & $\left(\left\{\theta_{i}^{*}\right\}_{i=0}^{d} ;\left\{\theta_{i}\right\}_{i=0}^{d} ;\left\{\varphi_{i}\right\}_{i=1}^{d} ;\left\{\phi_{d-i+1}\right\}_{i=1}^{d}\right)$ \\
$\Phi^{\downarrow *}$ & $\left(\left\{\theta_{d-i}^{*}\right\}_{i=0}^{d} ;\left\{\theta_{i}\right\}_{i=0}^{d} ;\left\{\phi_{d-i+1}\right\}_{i=1}^{d} ;\left\{\varphi_{i}\right\}_{i=1}^{d}\right)$ \\
$\Phi^{\Downarrow *}$ & $\left(\left\{\theta_{i}^{*}\right\}_{i=0}^{d} ;\left\{\theta_{d-i}\right\}_{i=0}^{d} ;\left\{\phi_{i}\right\}_{i=1}^{d} ;\left\{\varphi_{d-i+1}\right\}_{i=1}^{d}\right)$ \\
$\Phi^{\downarrow \Downarrow *}$ & $\left(\left\{\theta_{d-i}^{*}\right\}_{i=0}^{d} ;\left\{\theta_{d-i}\right\}_{i=0}^{d} ;\left\{\varphi_{d-i+1}\right\}_{i=1}^{d} ;\left\{\phi_{i}\right\}_{i=1}^{d}\right)$
\end{tabular}

\section{Affine transformations of a Leonard system}

In this section we consider the affine transformations of a Leonard system. We start with an observation.

Lemma 5.1 Let $\Phi=\left(A ;\left\{E_{i}\right\}_{i=0}^{d} ; A^{*} ;\left\{E_{i}^{*}\right\}_{i=0}^{d}\right)$ denote a Leonard system in $\mathcal{A}$. Let $\xi, \zeta, \xi^{*}, \zeta^{*}$ denote scalars in $\mathbb{K}$ with $\xi, \xi^{*}$ nonzero. Then the sequence

$$
\left(\xi A+\zeta I ;\left\{E_{i}\right\}_{i=0}^{d} ; \xi^{*} A^{*}+\zeta^{*} I ;\left\{E_{i}^{*}\right\}_{i=0}^{d}\right)
$$

is a Leonard system in $\mathcal{A}$.

Definition 5.2 Referring to Lemma 5.1, we call (10) the affine transformation of $\Phi$ associated with $\xi, \zeta, \xi^{*}, \zeta^{*}$.

Definition 5.3 Let $\Phi$ and $\Phi^{\prime}$ denote Leonard systems over $\mathbb{K}$. We say $\Phi$ and $\Phi^{\prime}$ are affine isomorphic whenever $\Phi$ is isomorphic to an affine transformation of $\Phi^{\prime}$. Observe that affine isomorphism is an equivalence relation.

Let $\Phi$ denote a Leonard system. We now consider how the set of relatives of $\Phi$ is partitioned into affine isomorphism classes. In order to avoid trivialities we assume the diameter of $\Phi$ is at least 1. The following is our main result on this topic. 
Theorem 5.4 Let $\Phi$ denote a Leonard system with first split sequence $\left\{\varphi_{i}\right\}_{i=1}^{d}$ and second split sequence $\left\{\phi_{i}\right\}_{i=1}^{d}$. Assume $d \geq 1$.

(i) Assume $\varphi_{1}=\varphi_{d}=-\phi_{1}=-\phi_{d}$. Then all eight relatives of $\Phi$ are mutually affine isomorphic.

(ii) Assume $\varphi_{1}=\varphi_{d}, \phi_{1}=\phi_{d}$ and $\varphi_{1} \neq-\phi_{1}$. Then the relatives of $\Phi$ form exactly two affine isomorphism classes, consisting of $\left\{\Phi, \Phi^{\downarrow \Downarrow}, \Phi^{*}, \Phi^{\downarrow \Downarrow *}\right\},\left\{\Phi^{\downarrow}, \Phi^{\Downarrow}, \Phi^{\downarrow *}, \Phi^{\Downarrow *}\right\}$.

(iii) Assume $\varphi_{1}=\varphi_{d}$ and $\phi_{1} \neq \phi_{d}$. Then the relatives of $\Phi$ form exactly four affine isomorphism classes, consisting of $\left\{\Phi, \Phi^{\downarrow \Downarrow *}\right\},\left\{\Phi^{\downarrow}, \Phi^{\downarrow *}\right\},\left\{\Phi^{\Downarrow}, \Phi^{\Downarrow *}\right\},\left\{\Phi^{\downarrow \Downarrow}, \Phi^{*}\right\}$.

(iv) Assume $\phi_{1}=\phi_{d}$ and $\varphi_{1} \neq \varphi_{d}$. Then the relatives of $\Phi$ form exactly four affine isomorphism classes, consisting of $\left\{\Phi, \Phi^{*}\right\},\left\{\Phi^{\downarrow}, \Phi^{\Downarrow *}\right\},\left\{\Phi^{\Downarrow}, \Phi^{\downarrow *}\right\},\left\{\Phi^{\downarrow \Downarrow}, \Phi^{\downarrow \Downarrow *}\right\}$.

(v) Assume $\varphi_{1}=-\phi_{1}, \varphi_{d}=-\phi_{d}$ and $\varphi_{1} \neq \varphi_{d}$. Then the relatives of $\Phi$ form exactly four affine isomorphism classes, consisting of $\left\{\Phi, \Phi^{\Downarrow}\right\},\left\{\Phi^{\downarrow}, \Phi^{\downarrow \Downarrow}\right\},\left\{\Phi^{*}, \Phi^{\Downarrow *}\right\},\left\{\Phi^{\downarrow *}, \Phi^{\downarrow \Downarrow *}\right\}$.

(vi) Assume $\varphi_{1}=-\phi_{d}, \varphi_{d}=-\phi_{1}$ and $\varphi_{1} \neq \varphi_{d}$. Then the relatives of $\Phi$ form exactly four affine isomorphism classes, consisting of $\left\{\Phi, \Phi^{\downarrow}\right\},\left\{\Phi^{\Downarrow}, \Phi^{\downarrow \Downarrow}\right\},\left\{\Phi^{*}, \Phi^{\downarrow *}\right\},\left\{\Phi^{\Downarrow *}, \Phi^{\downarrow \downarrow *}\right\}$.

(vii) Assume none of (i)-(vi) hold above. Then $\varphi_{1} \neq \varphi_{d}, \phi_{1} \neq \phi_{d}$, at least one of $\varphi_{1} \neq$ $-\phi_{1}, \varphi_{d} \neq-\phi_{d}$, and at least one of $\varphi_{1} \neq-\phi_{d}, \varphi_{d} \neq-\phi_{1}$. In this case the eight relatives of $\Phi$ are mutually non affine isomorphic.

The proof of Theorem 5.4 will be given in Section 9. In Sections 6-8 we obtain some results that will be used in this proof.

\section{How the parameter array is affected by affine transforma- tion}

Let $\Phi$ denote a Leonard system. In this section we consider how the parameter array of $\Phi$ is affected by affine transformation.

Lemma 6.1 Referring to Lemma 5.1, let $\left(\left\{\theta_{i}\right\}_{i=0}^{d} ;\left\{\theta_{i}^{*}\right\}_{i=0}^{d} ;\left\{\varphi_{i}\right\}_{i=1}^{d} ;\left\{\phi_{i}\right\}_{i=1}^{d}\right)$ denote the parameter array of $\Phi$. Then the parameter array of the Leonard system (10) is

$$
\left(\left\{\xi \theta_{i}+\zeta\right\}_{i=0}^{d} ;\left\{\xi^{*} \theta_{i}^{*}+\zeta^{*}\right\}_{i=0}^{d} ;\left\{\xi \xi^{*} \varphi_{i}\right\}_{i=1}^{d} ;\left\{\xi \xi^{*} \phi_{i}\right\}_{i=1}^{d}\right) .
$$

Proof. By Definition 4.1, for $0 \leq i \leq d$ the scalar $\theta_{i}$ is the eigenvalue of $A$ associated with $E_{i}$, so $\xi \theta_{i}+\zeta$ is the eigenvalue of $\xi A+\zeta I$ associated with $E_{i}$. Thus $\left\{\xi \theta_{i}+\zeta\right\}_{i=0}^{d}$ is the eigenvalue sequence of (10). Similarly $\left\{\xi^{*} \theta_{i}^{*}+\zeta^{*}\right\}_{i=0}^{d}$ is the dual eigenvalue sequence of (10). In the right-hand side of (6), if we replace $A$ by $\xi A+\zeta I$, and if we replace $\theta_{j}, \theta_{j}^{*}$ by $\xi \theta_{j}+\zeta, \xi^{*} \theta_{j}^{*}+\zeta^{*}(0 \leq j \leq d)$ and simplify the result we get $\xi \xi^{*} \varphi_{i}$. Therefore $\left\{\xi \xi^{*} \varphi_{i}\right\}_{i=1}^{d}$ is the first split sequence of (10). Similarly $\left\{\xi \xi^{*} \phi_{i}\right\}_{i=1}^{d}$ is the second split sequence of (10) and the result follows. 


\section{Some equations} 5.4 .

In this section we obtain some equations that will be useful in the proof of Theorem

Notation 7.1 Let $\Phi=\left(A ;\left\{E_{i}\right\}_{i=0}^{d} ; A^{*} ;\left\{E_{i}^{*}\right\}_{i=0}^{d}\right)$ denote a Leonard system over $\mathbb{K}$, with parameter array $\left(\left\{\theta_{i}\right\}_{i=0}^{d} ;\left\{\theta_{i}^{*}\right\}_{i=0}^{d} ;\left\{\varphi_{i}\right\}_{i=1}^{d} ;\left\{\phi_{i}\right\}_{i=1}^{d}\right)$. To avoid trivialities we assume $d \geq 1$.

Lemma 7.2 [29, Lemma 9.5] Referring to Notation 7.1,

$$
\frac{\theta_{h}-\theta_{d-h}}{\theta_{0}-\theta_{d}}=\frac{\theta_{h}^{*}-\theta_{d-h}^{*}}{\theta_{0}^{*}-\theta_{d}^{*}} \quad(0 \leq h \leq d)
$$

Definition 7.3 Referring to Notation 7.1, for $1 \leq i \leq d$ we have

$$
\sum_{h=0}^{i-1} \frac{\theta_{h}-\theta_{d-h}}{\theta_{0}-\theta_{d}}=\sum_{h=0}^{i-1} \frac{\theta_{h}^{*}-\theta_{d-h}^{*}}{\theta_{0}^{*}-\theta_{d}^{*}} .
$$

We denote this common value by $\vartheta_{i}$. We observe that $\vartheta_{1}=1$ and $\vartheta_{i}=\vartheta_{d-i+1}$ for $1 \leq i \leq d$.

Lemma 7.4 Referring to Notation 7.1 and Definition 7.3, the following hold for $1 \leq i \leq d$.

$$
\begin{aligned}
\varphi_{i} & =\phi_{1} \vartheta_{i}+\left(\theta_{i}^{*}-\theta_{0}^{*}\right)\left(\theta_{i-1}-\theta_{d}\right), \\
\varphi_{d-i+1} & =\phi_{1} \vartheta_{i}+\left(\theta_{d-i+1}^{*}-\theta_{0}^{*}\right)\left(\theta_{d-i}-\theta_{d}\right), \\
\varphi_{i} & =\phi_{d} \vartheta_{i}+\left(\theta_{i}-\theta_{0}\right)\left(\theta_{i-1}^{*}-\theta_{d}^{*}\right), \\
\varphi_{d-i+1} & =\phi_{d} \vartheta_{i}+\left(\theta_{d-i+1}-\theta_{0}\right)\left(\theta_{d-i}^{*}-\theta_{d}^{*}\right), \\
\phi_{i} & =\varphi_{1} \vartheta_{i}+\left(\theta_{i}^{*}-\theta_{0}^{*}\right)\left(\theta_{d-i+1}-\theta_{0}\right), \\
\phi_{d-i+1} & =\varphi_{1} \vartheta_{i}+\left(\theta_{d-i+1}^{*}-\theta_{0}^{*}\right)\left(\theta_{i}-\theta_{0}\right), \\
\phi_{i} & =\varphi_{d} \vartheta_{i}+\left(\theta_{d-i}-\theta_{d}\right)\left(\theta_{i-1}^{*}-\theta_{d}^{*}\right), \\
\phi_{d-i+1} & =\varphi_{d} \vartheta_{i}+\left(\theta_{i-1}-\theta_{d}\right)\left(\theta_{d-i}^{*}-\theta_{d}^{*}\right) .
\end{aligned}
$$

Proof. Apply $D_{4}$ to the equation (PA3) from Theorem4.4, and use Lemma4.5,

\section{The relatives and affine transformations of a Leonard sys- tem}

Let $\Phi$ denote a Leonard system in $\mathcal{A}$. In this section we give, for each relative of $\Phi$, necessary and sufficient conditions for it to be affine isomorphic to $\Phi$. Recall that by Theorem 4.4, two Leonard systems are isomorphic if and only if they have the same parameter array.

Lemma 8.1 Let $\Phi$ and $\Phi^{\prime}$ denote Leonard systems over $\mathbb{K}$ which are affine isomorphic. Then $\Phi^{g}$ and $\Phi^{\prime g}$ are affine isomorphic for all $g \in D_{4}$.

Proof. Routine. 
Proposition 8.2 Referring to Notation 7.1, let $\xi, \zeta, \xi^{*}, \zeta^{*}$ denote scalars in $\mathbb{K}$ with $\xi, \xi^{*}$ nonzero. Then $\Phi$ is isomorphic to the Leonard system (10) if and only if $\xi=1, \zeta=0$, $\xi^{*}=1, \zeta^{*}=0$.

Proof. Suppose that $\Phi$ is isomorphic to the Leonard system (10). Then these Leonard systems have the same parameter array. These parameter arrays are given in Notation 7.1 and (11); comparing them we find $\xi \theta_{i}+\zeta=\theta_{i}$ for $0 \leq i \leq d$. Setting $i=0, i=1$ in this equation we find $\xi=1, \zeta=0$. Similarly we find $\xi^{*}=1, \zeta^{*}=0$. This proves the result in one direction and the other direction is clear.

Lemma 8.3 Referring to Notation 7.1, let $\xi, \zeta, \xi^{*}, \zeta^{*}$ denote scalars in $\mathbb{K}$ with $\xi, \xi^{*}$ nonzero. Then $\Phi^{\downarrow}$ is isomorphic to the Leonard system (10) if and only if

$$
\begin{aligned}
\theta_{i} & =\xi \theta_{i}+\zeta & & (0 \leq i \leq d), \\
\theta_{d-i}^{*} & =\xi^{*} \theta_{i}^{*}+\zeta^{*} & & (0 \leq i \leq d), \\
\phi_{d-i+1} & =\xi \xi^{*} \varphi_{i} & & (1 \leq i \leq d), \\
\varphi_{d-i+1} & =\xi \xi^{*} \phi_{i} & & (1 \leq i \leq d) .
\end{aligned}
$$

Proof. Compare the parameter array of $\Phi^{\downarrow}$ from Lemma 4.5, with the parameter array (11).

Proposition 8.4 Referring to Notation 7.1, the following (i)-(iii) are equivalent.

(i) $\Phi^{\downarrow}$ is affine isomorphic to $\Phi$.

(ii) $\varphi_{1}=-\phi_{d}$ and $\varphi_{d}=-\phi_{1}$.

(iii) $\varphi_{i}=-\phi_{d-i+1}$ for $1 \leq i \leq d$ and $\theta_{i}^{*}+\theta_{d-i}^{*}$ is independent of $i$ for $0 \leq i \leq d$.

Suppose (i)-(iii) hold. Then $\Phi^{\downarrow}$ is isomorphic to (10) with $\xi=1, \zeta=0, \xi^{*}=-1$, and $\zeta^{*}$ equal to the common value of $\theta_{i}^{*}+\theta_{d-i}^{*}$.

Proof. $\quad(\mathrm{i}) \Rightarrow\left(\right.$ ii): By Definition 5.3 there exist scalars $\xi, \zeta, \xi^{*}, \zeta^{*}$ in $\mathbb{K}$ with $\xi, \xi^{*}$ nonzero such that $\Phi^{\downarrow}$ is isomorphic to the Leonard system (10). Now (20)-(23) hold by Lemma 8.3. Setting $i=0, i=1$ in (20) we find $\xi=1, \zeta=0$. Setting $i=0, i=d$ in (21) we find $\xi^{*}=-1$. Setting $i=1, i=d$ in (22) and using $\xi=1, \xi^{*}=-1$ we find $\varphi_{1}=-\phi_{d}$ and $\varphi_{d}=-\phi_{1}$.

(ii) $\Rightarrow\left(\right.$ iii): By (12), (19) and $\varphi_{d}=-\phi_{1}$,

$$
\varphi_{i}+\phi_{d-i+1}=\left(\theta_{i-1}-\theta_{d}\right)\left(\theta_{i}^{*}+\theta_{d-i}^{*}-\theta_{0}^{*}-\theta_{d}^{*}\right) \quad(1 \leq i \leq d) .
$$

By (14), (17) and $\varphi_{1}=-\phi_{d}$,

$$
\varphi_{i}+\phi_{d-i+1}=\left(\theta_{i}-\theta_{0}\right)\left(\theta_{i-1}^{*}+\theta_{d-i+1}^{*}-\theta_{0}^{*}-\theta_{d}^{*}\right) \quad(1 \leq i \leq d) .
$$

Replacing $i$ by $i+1$ in (25) and comparing the result with (24) we find

$$
\frac{\varphi_{i}+\phi_{d-i+1}}{\theta_{i-1}-\theta_{d}}=\frac{\varphi_{i+1}+\phi_{d-i}}{\theta_{i+1}-\theta_{0}} \quad(1 \leq i \leq d-1) .
$$


From this and since $\varphi_{1}+\phi_{d}=0$ we find $\varphi_{i}+\phi_{d-i+1}=0$ for $1 \leq i \leq d$. Evaluating (24) using this we find $\theta_{i}^{*}+\theta_{d-i}^{*}$ is independent of $i$ for $0 \leq i \leq d$.

(iii) $\Rightarrow(\mathrm{i})$ : Let $\zeta^{*}$ denote the common value of $\theta_{i}^{*}+\theta_{d-i}^{*}$, and let $\xi=1, \zeta=0, \xi^{*}=-1$. Now (20)-(23) hold so $\Phi^{\downarrow}$ is isomorphic to (10) by Lemma 8.3. Now $\Phi^{\downarrow}$ is affine isomorphic to $\Phi$ in view of Definition 5.3 ,

Proposition 8.5 Referring to Notation 7.1, the following (i)-(iii) are equivalent.

(i) $\Phi^{\Downarrow}$ is affine isomorphic to $\Phi$.

(ii) $\varphi_{1}=-\phi_{1}$ and $\varphi_{d}=-\phi_{d}$.

(iii) $\varphi_{i}=-\phi_{i}$ for $1 \leq i \leq d$ and $\theta_{i}+\theta_{d-i}$ is independent of $i$ for $0 \leq i \leq d$.

Suppose (i)-(iii) hold. Then $\Phi^{\Downarrow}$ is isomorphic to (10) with $\xi=-1$, $\zeta$ equal to the common value of $\theta_{i}+\theta_{d-i}, \xi^{*}=1$, and $\zeta^{*}=0$.

Proof. By Lemma 8.1 (with $g=*$ ) and since $\Downarrow *=* \downarrow$ we find $\Phi^{\Downarrow}$ is affine isomorphic to $\Phi$ if and only if $\Phi^{* \downarrow}$ is affine isomorphic to $\Phi^{*}$. Now apply Proposition 8.4 to $\Phi^{*}$ and use Lemma 4.5

Lemma 8.6 Referring to Notation 7.1, let $\xi, \zeta, \xi^{*}, \zeta^{*}$ denote scalars in $\mathbb{K}$ with $\xi, \xi^{*}$ nonzero. Then $\Phi^{*}$ is isomorphic to the Leonard system (10) if and only if

$$
\begin{aligned}
\theta_{i}^{*} & =\xi \theta_{i}+\zeta & & (0 \leq i \leq d), \\
\theta_{i} & =\xi^{*} \theta_{i}^{*}+\zeta^{*} & & (0 \leq i \leq d), \\
\varphi_{i} & =\xi \xi^{*} \varphi_{i} & & (1 \leq i \leq d), \\
\phi_{d-i+1} & =\xi \xi^{*} \phi_{i} & & (1 \leq i \leq d) .
\end{aligned}
$$

Proof. Compare the parameter array of $\Phi^{*}$ from Lemma 4.5, with the parameter array (11).

Proposition 8.7 Referring to Notation 7.1, the following (i)-(iv) are equivalent.

(i) $\Phi^{*}$ is affine isomorphic to $\Phi$.

(ii) $\phi_{1}=\phi_{d}$.

(iii) $\phi_{i}=\phi_{d-i+1}$ for $1 \leq i \leq d$.

(iv) $\left(\theta_{i}^{*}-\theta_{0}^{*}\right)\left(\theta_{i}-\theta_{0}\right)^{-1}$ is independent of $i$ for $1 \leq i \leq d$.

Suppose (i)-(iv) hold. Then $\Phi^{*}$ is isomorphic to (10) with $\xi$ equal to the common value of $\left(\theta_{i}^{*}-\theta_{0}^{*}\right)\left(\theta_{i}-\theta_{0}\right)^{-1}, \zeta=\theta_{0}^{*}-\xi \theta_{0}, \xi^{*}=\xi^{-1}$, and $\zeta^{*}=\theta_{0}-\xi^{*} \theta_{0}^{*}$.

Proof. (i) $\Rightarrow$ (ii): By Definition 5.3 there exist scalars $\xi, \zeta, \xi^{*}, \zeta^{*}$ in $\mathbb{K}$ with $\xi, \xi^{*}$ nonzero such that $\Phi^{*}$ is isomorphic to the Leonard system (10). Now (26)-(29) hold by Lemma 8.6. By (28) we find $\xi \xi^{*}=1$. Setting $i=1$ in (29) and using $\xi \xi^{*}=1$ we find $\phi_{1}=\phi_{d}$. 
(ii) $\Rightarrow$ (iv): For $0 \leq i \leq d$ define $\eta_{i}=\left(\theta_{i}^{*}-\theta_{0}^{*}\right)\left(\theta_{d}-\theta_{0}\right)-\left(\theta_{i}-\theta_{0}\right)\left(\theta_{d}^{*}-\theta_{0}^{*}\right)$ and observe $\eta_{0}=0$. We show $\eta_{i}=0$ for $1 \leq i \leq d$. By (12), (14) and since $\phi_{1}=\phi_{d}$,

$$
\left(\theta_{i}^{*}-\theta_{0}^{*}\right)\left(\theta_{i-1}-\theta_{d}\right)=\left(\theta_{i}-\theta_{0}\right)\left(\theta_{i-1}^{*}-\theta_{d}^{*}\right) \quad(1 \leq i \leq d) .
$$

In this equation we rearrange terms to get

$$
\eta_{i}\left(\theta_{i-1}-\theta_{d}\right)=\eta_{i-1}\left(\theta_{i}-\theta_{0}\right) \quad(1 \leq i \leq d)
$$

By this and since $\eta_{0}=0$ we find $\eta_{i}=0$ for $1 \leq i \leq d$. The result follows.

(iv) $\Rightarrow\left(\right.$ iii): Let $i$ be given. Since $\left(\theta_{i}^{*}-\theta_{0}^{*}\right)\left(\theta_{i}-\theta_{0}\right)^{-1}$ is independent of $i$,

$$
\left(\theta_{i}^{*}-\theta_{0}^{*}\right)\left(\theta_{d-i+1}-\theta_{0}\right)=\left(\theta_{d-i+1}^{*}-\theta_{0}^{*}\right)\left(\theta_{i}-\theta_{0}\right) .
$$

Comparing (16) and (17) using this we find $\phi_{i}=\phi_{d-i+1}$.

(iii) $\Rightarrow$ (ii): Clear.

(iii), (iv) $\Rightarrow$ (i): Let $\xi$ denote the common value of $\left(\theta_{i}^{*}-\theta_{0}^{*}\right)\left(\theta_{i}-\theta_{0}\right)^{-1}$ and set $\xi^{*}=\xi^{-1}$, $\zeta=\theta_{0}^{*}-\xi \theta_{0}, \zeta^{*}=\theta_{0}-\xi^{*} \theta_{0}^{*}$. Then (26)-(29) hold so $\Phi^{*}$ is isormorphic to (10) by Lemma 8.6. Now $\Phi^{*}$ is affine isomorphic to $\Phi$ in view of Definition 5.3 .

Proposition 8.8 Referring to Notation 7.1, the following (i)-(iv) are equivalent.

(i) $\Phi^{\downarrow \Downarrow *}$ is affine isomorphic to $\Phi$.

(ii) $\varphi_{1}=\varphi_{d}$.

(iii) $\varphi_{i}=\varphi_{d-i+1}$ for $1 \leq i \leq d$.

(iv) $\left(\theta_{d-i}^{*}-\theta_{d}^{*}\right)\left(\theta_{i}-\theta_{0}\right)^{-1}$ is independent of $i$ for $1 \leq i \leq d$.

Suppose (i)-(iv) hold. Then $\Phi^{\downarrow \Downarrow *}$ is isomorphic to (10) with $\xi$ equal to the common value of $\left(\theta_{d-i}^{*}-\theta_{d}^{*}\right)\left(\theta_{i}-\theta_{0}\right)^{-1}, \zeta=\theta_{d}^{*}-\xi \theta_{0}, \xi^{*}=\xi^{-1}$, and $\zeta^{*}=\theta_{0}-\xi^{*} \theta_{d}^{*}$.

Proof. By Lemma 8.1 (with $g=\downarrow$ ) and since $\Downarrow * \downarrow=*$ we find that $\Phi^{\downarrow \Downarrow *}$ is affine isomorphic to $\Phi$ if and only if $\Phi^{\downarrow *}$ is affine isomorphic to $\Phi^{\downarrow}$. Now apply Proposition 8.7 to $\Phi^{\downarrow}$ and use Lemma 4.5 ,

Lemma 8.9 Referring to Notation 7.1, let $\xi, \zeta, \xi^{*}, \zeta^{*}$ denote scalars in $\mathbb{K}$ with $\xi, \xi^{*}$ nonzero. Then $\Phi^{\downarrow \Downarrow}$ is isomorphic to the Leonard system (10) if and only if

$$
\begin{aligned}
\theta_{d-i} & =\xi \theta_{i}+\zeta & & (0 \leq i \leq d), \\
\theta_{d-i}^{*} & =\xi^{*} \theta_{i}^{*}+\zeta^{*} & & (0 \leq i \leq d), \\
\varphi_{d-i+1} & =\xi \xi^{*} \varphi_{i} & & (1 \leq i \leq d), \\
\phi_{d-i+1} & =\xi \xi^{*} \phi_{i} & & (1 \leq i \leq d) .
\end{aligned}
$$

Proof. Compare the parameter array of $\Phi^{\downarrow \Downarrow}$ from Lemma 4.5, with the parameter array (11). 
Proposition 8.10 Referring to Notation 7.1, the following (i)-(iv) are equivalent.

(i) $\Phi^{\downarrow \Downarrow}$ is affine isomorphic to $\Phi$.

(ii) $\varphi_{1}=\varphi_{d}$ and $\phi_{1}=\phi_{d}$.

(iii) $\varphi_{i}=\varphi_{d-i+1}$ and $\phi_{i}=\phi_{d-i+1}$ for $1 \leq i \leq d$.

(iv) Each of $\left(\theta_{i}^{*}-\theta_{0}^{*}\right)\left(\theta_{i}-\theta_{0}\right)^{-1},\left(\theta_{d-i}^{*}-\theta_{d}^{*}\right)\left(\theta_{i}-\theta_{0}\right)^{-1}$ is independent of $i$ for $1 \leq i \leq d$. Suppose (i)-(iv) hold. Then each of $\theta_{i}+\theta_{d-i}, \theta_{i}^{*}+\theta_{d-i}^{*}$ is independent of $i$ for $0 \leq i \leq d$. Moreover $\Phi^{\downarrow \Downarrow}$ is isomorphic to (10) with $\xi=-1, \xi^{*}=-1$, and $\zeta$ (resp. $\zeta^{*}$ ) equal to the common value of $\theta_{i}+\theta_{d-i}\left(\right.$ resp. $\left.\theta_{i}^{*}+\theta_{d-i}^{*}\right)$.

Proof. (i) $\Rightarrow$ (ii): By Definition 5.3 there exist scalars $\xi, \zeta, \xi^{*}, \zeta^{*}$ in $\mathbb{K}$ with $\xi, \xi^{*}$ nonzero such that $\Phi^{\downarrow \Downarrow}$ is isomorphic to the Leonard system (10). Now (30)-(33) hold by Lemma 8.9. Setting $i=0, i=d$ in (30) we find $\xi=-1$. Setting $i=0, i=d$ in (31) we find $\xi^{*}=-1$. Setting $i=1$ in (32), (33) and using $\xi=-1, \xi^{*}=-1$ we find $\varphi_{1}=\varphi_{d}$ and $\phi_{1}=\phi_{d}$.

(ii) $\Leftrightarrow($ iii $) \Leftrightarrow($ iv $)$ : Follows from Propositions 8.7 and 8.8 .

(iii), (iv) $\Rightarrow$ (i): We first show that $\theta_{i}^{*}+\theta_{d-i}^{*}$ is independent of $i$ for $0 \leq i \leq d$. By assumption

and

$$
\frac{\theta_{i}^{*}-\theta_{0}^{*}}{\theta_{i}-\theta_{0}}=\frac{\theta_{d}^{*}-\theta_{0}^{*}}{\theta_{d}-\theta_{0}} \quad(1 \leq i \leq d)
$$

$$
\frac{\theta_{d-i}^{*}-\theta_{d}^{*}}{\theta_{i}-\theta_{0}}=\frac{\theta_{0}^{*}-\theta_{d}^{*}}{\theta_{d}-\theta_{0}} \quad(1 \leq i \leq d) .
$$

Adding (34), (35) we find $\theta_{i}^{*}+\theta_{d-i}^{*}=\theta_{0}^{*}+\theta_{d}^{*}$ for $1 \leq i \leq d$. Therefore $\theta_{i}^{*}+\theta_{d-i}^{*}$ is independent of $i$ for $0 \leq i \leq d$. Next we show that $\theta_{i}+\theta_{d-i}$ is independent of $i$ for $0 \leq i \leq d$. Rearranging the terms in (34) we find that for $1 \leq i \leq d$,

$$
\frac{\theta_{i}+\theta_{d-i}-\theta_{0}-\theta_{d}}{\theta_{0}-\theta_{d}}=\frac{\theta_{i}^{*}+\theta_{d-i}^{*}-\theta_{0}^{*}-\theta_{d}^{*}}{\theta_{0}^{*}-\theta_{d}^{*}} .
$$

In the above equation the numerator on the right is zero so the numerator on the left is zero. Therefore $\theta_{i}+\theta_{d-i}$ is independent of $i$ for $0 \leq i \leq d$. Now let $\zeta$ (resp. $\zeta^{*}$ ) denote the common value of $\theta_{i}+\theta_{d-i}$ (resp. $\left.\theta_{i}^{*}+\theta_{d-i}^{*}\right)$, and let $\xi=-1, \xi^{*}=-1$. Then (30)-(33) hold so $\Phi^{\downarrow \Downarrow}$ is isomorpic to (10) by Lemma 8.9. Now $\Phi^{\downarrow \Downarrow}$ is affine isomorphic to $\Phi$ in view of Definition 5.3 .

Lemma 8.11 Referring to Notation 7.1, let $\xi, \zeta, \xi^{*}, \zeta^{*}$ denote scalars in $\mathbb{K}$ with $\xi, \xi^{*}$ nonzero. Then $\Phi^{\downarrow *}$ is isomorphic to the Leonard system (10) if and only if

$$
\begin{aligned}
\theta_{d-i}^{*} & =\xi \theta_{i}+\zeta & & (0 \leq i \leq d), \\
\theta_{i} & =\xi^{*} \theta_{i}^{*}+\zeta^{*} & & (0 \leq i \leq d), \\
\phi_{d-i+1} & =\xi \xi^{*} \varphi_{i} & & (1 \leq i \leq d), \\
\varphi_{i} & =\xi \xi^{*} \phi_{i} & & (1 \leq i \leq d) .
\end{aligned}
$$

Proof. Compare the parameter array of $\Phi^{\downarrow *}$ from Lemma 4.5, with the parameter array (11). 
Proposition 8.12 Referring to Notation 7.1, the following (i)-(iii) are equivalent.

(i) $\Phi^{\downarrow *}$ is affine isomorphic to $\Phi$.

(ii) $\varphi_{1}=\varphi_{d}=-\phi_{1}=-\phi_{d}$.

(iii) $\varphi_{i}, \varphi_{d-i+1},-\phi_{i},-\phi_{d-i+1}$ coincide for $1 \leq i \leq d$ and each of $\left(\theta_{i}^{*}-\theta_{0}^{*}\right)\left(\theta_{i}-\theta_{0}\right)^{-1}$, $\left(\theta_{d-i}^{*}-\theta_{d}^{*}\right)\left(\theta_{i}-\theta_{0}\right)^{-1}$ is independent of $i$ for $1 \leq i \leq d$.

Suppose (i)-(iii) hold. Then $\Phi^{\downarrow *}$ is isomorphic to (10) with $\xi$ equal to the common value of $\left(\theta_{d-i}^{*}-\theta_{d}^{*}\right)\left(\theta_{i}-\theta_{0}\right)^{-1}, \zeta=\theta_{d}^{*}-\xi \theta_{0}, \xi^{*}=-\xi^{-1}$, and $\zeta^{*}=\theta_{0}-\xi^{*} \theta_{0}^{*}$.

Proof. $\quad(\mathrm{i}) \Rightarrow\left(\right.$ ii): By Definition 5.3 there exist scalars $\xi, \zeta, \xi^{*}, \zeta^{*}$ in $\mathbb{K}$ with $\xi, \xi^{*}$ nonzero such that $\Phi^{\downarrow *}$ is isomorphic to the Leonard system (10). Now (36)-(39) hold by Lemma 8.11. Setting $i=0, i=d$ in (36) $)$ we find $\xi\left(\theta_{0}-\theta_{d}\right)=\theta_{d}^{*}-\theta_{0}^{*}$. Setting $i=0, i=d$ in (37) we find $\xi^{*}\left(\theta_{0}^{*}-\theta_{d}^{*}\right)=\theta_{0}-\theta_{d}$. By these comments $\xi \xi^{*}=-1$. Setting $i=1, i=d$ in (38) and using $\xi \xi^{*}=-1$ we find $\varphi_{1}=-\phi_{d}$ and $\varphi_{d}=-\phi_{1}$. Setting $i=1$ in (39) and using $\xi \xi^{*}=-1$ we find $\varphi_{1}=-\phi_{1}$.

(ii) $\Leftrightarrow$ (iii): Follows from Propositions 8.4, 8.5 and 8.10,

(ii), (iii) $\Rightarrow$ (i): Let $\xi$ denote the common value of $\left(\theta_{d-i}^{*}-\theta_{d}^{*}\right)\left(\theta_{i}-\theta_{0}\right)^{-1}$, and let $\xi^{*}=-\xi^{-1}$, $\zeta=\theta_{d}^{*}-\xi \theta_{0}, \zeta^{*}=\theta_{0}-\xi^{*} \theta_{0}^{*}$. Then (36) $-(39)$ hold so $\Phi^{\downarrow *}$ is isormorphic to (10) by Lemma 8.11, Now $\Phi^{\downarrow *}$ is affine isomorphic to $\Phi$ in view of Definition 5.3 .

Proposition 8.13 Referring to Notation [7.1, the following (i)-(iii) are equivalent.

(i) $\Phi^{\Downarrow *}$ is affine isomorphic to $\Phi$.

(ii) $\varphi_{1}=\varphi_{d}=-\phi_{1}=-\phi_{d}$.

(iii) $\varphi_{i}, \varphi_{d-i+1},-\phi_{i},-\phi_{d-i+1}$ coincide for $1 \leq i \leq d$ and each of $\left(\theta_{i}^{*}-\theta_{0}^{*}\right)\left(\theta_{i}-\theta_{0}\right)^{-1}$, $\left(\theta_{d-i}^{*}-\theta_{d}^{*}\right)\left(\theta_{i}-\theta_{0}\right)^{-1}$ is independent of $i$ for $1 \leq i \leq d$.

Suppose (i)-(iii) hold. Then $\Phi^{\Downarrow *}$ is affine isomorphic to (10) with $\xi$ equal to the common value of $\left(\theta_{i}^{*}-\theta_{0}^{*}\right)\left(\theta_{i}-\theta_{0}\right)^{-1}, \zeta=\theta_{0}^{*}-\xi \theta_{0}, \xi^{*}=-\xi^{-1}$, and $\zeta^{*}=\theta_{0}-\xi^{*} \theta_{d}^{*}$.

Proof. By Lemma 8.1 (with $g=\downarrow$ ) and since $\Downarrow * \downarrow=*=\downarrow \downarrow *$ we find that $\Phi^{\Downarrow *}$ is affine isomorphic to $\Phi$ if and only if $\left(\Phi^{\downarrow}\right)^{\downarrow *}$ is affine isomorphic to $\Phi^{\downarrow}$. Now apply Proposition 8.12 to $\Phi^{\downarrow}$ and use Lemma 4.5 .

\section{Proof of Theorem 5.4}

In this section we prove Theorem 5.4 .

Proof of Theorem 5.4. (i): Observe that $\Phi^{g}$ is isomorphic to $\Phi$ for all $g \in D_{4}$ by Propositions 8.4, 8.5, 8.7, 8.8, 8.10, 8.12 and 8.13,

(ii): Since $\varphi_{1}=\varphi_{d}$ and $\phi_{1}=\phi_{d}$, the Leonard systems $\Phi^{*}, \Phi^{\downarrow \Downarrow *}, \Phi^{\downarrow \Downarrow}$ are affine isomorphic to $\Phi$ by Propositions 8.7, 8.8, 8.10 respectively. Therefore $\Phi, \Phi^{*}, \Phi^{\downarrow \downarrow *}, \Phi^{\downarrow \Downarrow}$ are contained in a common affine isomorphism class. By this and Lemma 8.1 the Leonard 
systems $\Phi^{\downarrow}, \Phi^{\Downarrow}, \Phi^{\downarrow *}, \Phi^{\Downarrow *}$ are contained in a common isomorphism class. The above affine isomorphism classes are distinct; indeed $\Phi^{\downarrow}$ is not affine isomorphic to $\Phi$ by Proposition 8.4 and since $\varphi_{1} \neq-\phi_{d}$. The result follows.

(iii): By Propostion 8.8 the Leonard system $\Phi$ is affine isomorphic to $\Phi^{\downarrow \downarrow *}$. By Propositions $8.4,8.5,8.10,8.7,8.12,8.13$, $\Phi$ is not affine isomorphic to any of $\Phi^{\downarrow}, \Phi^{\Downarrow}, \Phi^{\downarrow \Downarrow}, \Phi^{*}$, $\Phi^{\downarrow *}, \Phi^{\Downarrow *}$. The result follows from these comments in view of Lemma 8.1 and (44), (5)).

(iv): By Propostion 8.7 the Leonard system $\Phi$ is affine isomorphic to $\Phi^{*}$. By Propositions 8.4, 8.5, 8.10, 8.12, 8.13, 8.8, $\Phi$ is not affine isomorphic to any of $\Phi^{\downarrow}, \Phi^{\Downarrow}, \Phi^{\downarrow \Downarrow}, \Phi^{\downarrow *}$, $\Phi^{\Downarrow *}, \Phi^{\downarrow \Downarrow *}$. The result follows from these comments in view of Lemma 8.1 and (44), (5).

(v): By Propostion 8.5 the Leonard system $\Phi$ is affine isomorphic to $\Phi^{\Downarrow}$. By Propositions 8.4, 8.10, 8.7, 8.12, 8.13, 8.8, $\Phi$ is not affine isomorphic to any of $\Phi^{\downarrow}, \Phi^{\downarrow \Downarrow}, \Phi^{*}, \Phi^{\downarrow *}$, $\Phi^{\Downarrow *}, \Phi^{\downarrow \Downarrow *}$. The result follows from these comments in view of Lemma 8.1 and (44), (5).

(vi): By Propostion 8.4 the Leonard system $\Phi$ is affine isomorphic to $\Phi^{\downarrow}$. By Propositions 8.5, 8.10, 8.7, 8.12, 8.13, 8.8, $\Phi$ is not affine isomorphic to any of $\Phi^{\Downarrow}, \Phi^{\downarrow \Downarrow}, \Phi^{*}, \Phi^{\downarrow *}$, $\Phi^{\Downarrow *}, \Phi^{\downarrow \Downarrow *}$. The result follows from these comments in view of Lemma 8.1 and (4), (5)).

(vii): By Propositions 8.4, 8.5, 8.7, 8.10, 8.12, 8.13, 8.8, $\Phi$ is not affine isomorphic to any of $\Phi^{\downarrow}, \Phi^{\Downarrow}, \Phi^{*}, \Phi^{\downarrow \Downarrow}, \Phi^{\downarrow *}, \Phi^{\Downarrow *}, \Phi^{\downarrow \Downarrow *}$. The result follows from this and Lemma 8.1 ,

\section{The parameters $a_{i}$ and $a_{i}^{*}$}

It turns out that for some of the cases of Theorem 5.4 there is a natural interpretation in terms of the parameters $a_{i}$ and $a_{i}^{*}$ [29, Definition 2.5]. In this section we explain the situation. We start with a definition.

Definition 10.1 29, Definition 2.5] Referring to Notation 7.1, for $0 \leq i \leq d$ we define scalars

$$
a_{i}:=\operatorname{tr}\left(E_{i}^{*} A\right), \quad a_{i}^{*}:=\operatorname{tr}\left(E_{i} A^{*}\right) .
$$

Lemma 10.2 Referring to Notation 7.1 and Definition 10.1, the following (i), (ii) are equaivalent.

(i) $a_{i}$ is independent of $i$ for $0 \leq i \leq d$.

(ii) The equivalent conditions (i)-(iii) hold in Proposition 8.5.

Suppose (i), (ii) hold. Then the common value of $\theta_{i}+\theta_{d-i}$ is twice the common value of $a_{i}$.

Proof. Follows from [18, Theorem 1.5] and Proposition 8.5. 
Lemma 10.3 Referring to Notation 7.1 and Definition 10.1, the following (i), (ii) are equaivalent.

(i) $a_{i}^{*}$ is independent of $i$ for $0 \leq i \leq d$.

(ii) The equivalent conditions (i)-(iii) hold in Proposition 8.4.

Suppose (i), (ii) hold. Then the common value of $\theta_{i}^{*}+\theta_{d-i}^{*}$ is twice the common value of $a_{i}^{*}$.

Proof. Follows from [18, Theorem 1.6] and Proposition 8.4 .

Theorem 10.4 Referring to Notation 7.1 and Definition 10.1, the following (i)-(iv) hold.

(i) In Case (i) of Theorem 5.4, each of $a_{i}, a_{i}^{*}$ is independent of $i$ for $0 \leq i \leq d$.

(ii) In Case (v) of Theorem 5.4, $a_{i}$ is independent of $i$ for $0 \leq i \leq d$ but $a_{i}^{*}$ is not independent of $i$ for $0 \leq i \leq d$.

(iii) In Case (vi) of Theorem 5.4, $a_{i}^{*}$ is independent of $i$ for $0 \leq i \leq d$ but $a_{i}$ is not independent of $i$ for $0 \leq i \leq d$.

(iv) In the remaining cases of Theorem 5.4, neither of $a_{i}$, $a_{i}^{*}$ is independent of $i$ for $0 \leq i \leq d$.

Proof. Follows from Theorem 5.4 and Lemmas 10.2, 10.3.

\section{Affine transformations of a Leonard pair}

Let $A, A^{*}$ denote a Leonard pair in $\mathcal{A}$ and let $\xi, \zeta, \xi^{*}, \zeta^{*}$ denote scalars in $\mathbb{K}$ with $\xi, \xi^{*}$ nonzero. By Lemma 5.1 and our comments below Definition 2.1 the pair

$$
\xi A+\zeta I, \quad \xi^{*} A^{*}+\zeta^{*} I
$$

is a Leonard pair in $\mathcal{A}$. We call (40) the affine transformation of $A, A^{*}$ associated with $\xi, \zeta, \xi^{*}, \zeta^{*}$. In this section we find necessary and sufficient conditions for the Leonard pair (40) to be isomorphic to $A, A^{*}$. We also find necessary and sufficient conditions for the Leonard pair (40) to be isomorphic to the Leonard pair $A^{*}, A$.

Notation 11.1 Let $A, A^{*}$ denote a Leonard pair in $\mathcal{A}$. Let $\Phi=\left(A ;\left\{E_{i}\right\}_{i=0}^{d} ; A^{*} ;\left\{E_{i}^{*}\right\}_{i=0}^{d}\right)$ denote a Leonard system associated with $A, A^{*}$ and let $\left(\left\{\theta_{i}\right\}_{i=0}^{d} ;\left\{\theta_{i}^{*}\right\}_{i=0}^{d} ;\left\{\varphi_{i}\right\}_{i=1}^{d} ;\left\{\phi_{i}\right\}_{i=1}^{d}\right)$ denote the parameter array of $\Phi$. To avoid trivialities we assume $d \geq 1$. 
Proposition 11.2 Referring to Notation 11.1, the Leonard pair $A, A^{*}$ is isomorphic to the Leonard pair (40) if and only if at least one of (i)-(iv) holds below.

(i) $\xi=1, \zeta=0, \xi^{*}=1, \zeta^{*}=0$.

(ii) $\varphi_{1}=-\phi_{d}, \varphi_{d}=-\phi_{1}, \xi=1, \zeta=0, \xi^{*}=-1, \zeta^{*}=\theta_{0}^{*}+\theta_{d}^{*}$.

(iii) $\varphi_{1}=-\phi_{1}, \varphi_{d}=-\phi_{d}, \xi=-1, \zeta=\theta_{0}+\theta_{d}, \xi^{*}=1, \zeta^{*}=0$.

(iv) $\varphi_{1}=\varphi_{d}, \phi_{1}=\phi_{d}, \xi=-1, \zeta=\theta_{0}+\theta_{d}, \xi^{*}=-1, \zeta^{*}=\theta_{0}^{*}+\theta_{d}^{*}$

In this case precisely one of (i)-(iv) holds.

Proof. By [32, Lemma 5.4] the Leonard systems associated with $A, A^{*}$ are $\Phi, \Phi^{\downarrow}, \Phi^{\Downarrow}$, $\Phi^{\downarrow \Downarrow}$. Therefore the Leonard pair $A, A^{*}$ is isomorphic to the Leonard pair (40) if and only if at least one of $\Phi, \Phi^{\downarrow}, \Phi^{\Downarrow}, \Phi^{\downarrow \Downarrow}$ is isomorphic to the Leonard system (10). By this and Propositions 8.2, 8.4, 8.5, 8.10 we find $A, A^{*}$ is isomorphic to (40) if and only if at least one of (i)-(iv) holds. Assume that at least one of (i)-(iv) holds. We show that precisely one of (i)-(iv) holds. By way of contradiction assume that at least two of (i)-(iv) hold. Then at least one of $2 \xi, 2 \xi^{*}$ is zero, forcing $\operatorname{Char}(\mathbb{K})=2$, and at least one of $\theta_{0}+\theta_{d}, \theta_{0}^{*}+\theta_{d}^{*}$ is zero, forcing $\operatorname{Char}(\mathbb{K}) \neq 2$ and giving a contradiction. Therefore precisely one of (i)-(iv) holds.

Proposition 11.3 Referring to Notation 11.1, the Leonard pair $A^{*}, A$ is isomorphic to the Leonard pair (40) if and only if at least one of (i)-(iv) holds below.

(i) $\phi_{1}=\phi_{d}, \xi=\left(\theta_{d}^{*}-\theta_{0}^{*}\right)\left(\theta_{d}-\theta_{0}\right)^{-1}, \zeta=\theta_{0}^{*}-\xi \theta_{0}, \xi^{*}=\xi^{-1}, \zeta^{*}=\theta_{0}-\xi^{*} \theta_{0}^{*}$.

(ii) $\varphi_{1}=\varphi_{d}=-\phi_{1}=-\phi_{d}, \xi=\left(\theta_{0}^{*}-\theta_{d}^{*}\right)\left(\theta_{d}-\theta_{0}\right)^{-1}, \zeta=\theta_{d}^{*}-\xi \theta_{0}, \xi^{*}=-\xi^{-1}$, $\zeta^{*}=\theta_{0}-\xi^{*} \theta_{0}^{*}$.

(iii) $\varphi_{1}=\varphi_{d}=-\phi_{1}=-\phi_{d}, \xi=\left(\theta_{d}^{*}-\theta_{0}^{*}\right)\left(\theta_{d}-\theta_{0}\right)^{-1}, \zeta=\theta_{0}^{*}-\xi \theta_{0}, \xi^{*}=-\xi^{-1}$, $\zeta^{*}=\theta_{0}-\xi^{*} \theta_{d}^{*}$

(iv) $\varphi_{1}=\varphi_{d}, \xi=\left(\theta_{0}^{*}-\theta_{d}^{*}\right)\left(\theta_{d}-\theta_{0}\right)^{-1}, \zeta=\theta_{d}^{*}-\xi \theta_{0}, \xi^{*}=\xi^{-1}, \zeta^{*}=\theta_{0}-\xi^{*} \theta_{d}^{*}$.

In this case precisely one of (i)-(iv) holds.

Proof. By [32, Lemma 5.4] the Leonard systems associated with $A^{*}, A$ are $\Phi^{*}, \Phi^{\downarrow *}, \Phi^{\Downarrow *}$, $\Phi^{\downarrow \Downarrow *}$. Therefore the Leonard pair $A^{*}, A$ is isomorphic to the Leonard pair (40) if and only if at least one of $\Phi^{*}, \Phi^{\downarrow *}, \Phi^{\Downarrow *}, \Phi^{\downarrow \Downarrow *}$ is isomorphic to the Leonard system (10). By this and Propositions 8.7, 8.8, 8.12, 8.13 we find $A^{*}, A$ is isomorphic to (40) if and only if at least one of (i)-(iv) holds. Assume that at least one of (i)-(iv) holds. We show that precisely one of (i)-(iv) holds. By way of contradiction assume that at least two of (i)-(iv) hold. Then at least one of $\theta_{0}=\theta_{d}, \theta_{0}^{*}=\theta_{d}^{*}$ holds, for a contradiction. Therefore precisely one of (i)-(iv) holds.

The following is the main result of the paper. 
Theorem 11.4 Referring to Notation 11.1, we set $\alpha=\left(\theta_{d}^{*}-\theta_{0}^{*}\right)\left(\theta_{d}-\theta_{0}\right)^{-1}$.

(i) Assume $\varphi_{1}=\varphi_{d}=-\phi_{1}=-\phi_{d}$. Then $A, A^{*}$ is isomorphic to (40) if and only if the sequence $\xi, \zeta, \xi^{*}, \zeta^{*}$ is listed in the following table.

\begin{tabular}{cccc}
$\xi$ & $\zeta$ & $\xi^{*}$ & $\zeta^{*}$ \\
\hline 1 & 0 & 1 & 0 \\
1 & 0 & -1 & $\theta_{0}^{*}+\theta_{d}^{*}$ \\
-1 & $\theta_{0}+\theta_{d}$ & 1 & 0 \\
-1 & $\theta_{0}+\theta_{d}$ & -1 & $\theta_{0}^{*}+\theta_{d}^{*}$
\end{tabular}

Moreover $A^{*}, A$ is isomorphic to (40) if and only if the sequence $\xi, \zeta, \xi^{*}, \zeta^{*}$ is listed in the following table.

\begin{tabular}{cccc}
$\xi$ & $\zeta$ & $\xi^{*}$ & $\zeta^{*}$ \\
\hline$\alpha$ & $\theta_{0}^{*}-\alpha \theta_{0}$ & $\alpha^{-1}$ & $\theta_{0}-\alpha^{-1} \theta_{0}^{*}$ \\
$-\alpha$ & $\theta_{d}^{*}+\alpha \theta_{0}$ & $\alpha^{-1}$ & $\theta_{0}-\alpha^{-1} \theta_{0}^{*}$ \\
$\alpha$ & $\theta_{0}^{*}-\alpha \theta_{0}$ & $-\alpha^{-1}$ & $\theta_{0}+\alpha^{-1} \theta_{d}^{*}$ \\
$-\alpha$ & $\theta_{d}^{*}+\alpha \theta_{0}$ & $-\alpha^{-1}$ & $\theta_{0}+\alpha^{-1} \theta_{d}^{*}$
\end{tabular}

(ii) Assume $\varphi_{1}=\varphi_{d}, \phi_{1}=\phi_{d}$ and $\varphi_{1} \neq-\phi_{1}$. Then $A, A^{*}$ is isomorphic to 40) if and only if the sequence $\xi, \zeta, \xi^{*}, \zeta^{*}$ is listed in the following table.

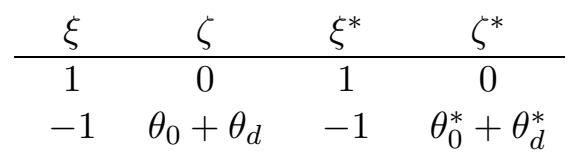

Moreover $A^{*}, A$ is isomorphic to (40) if and only if the sequence $\xi, \zeta, \xi^{*}, \zeta^{*}$ is listed in the following table.

\begin{tabular}{cccc}
$\xi$ & $\zeta$ & $\xi^{*}$ & $\zeta^{*}$ \\
\hline$\alpha$ & $\theta_{0}^{*}-\alpha \theta_{0}$ & $\alpha^{-1}$ & $\theta_{0}-\alpha^{-1} \theta_{0}^{*}$ \\
$-\alpha$ & $\theta_{d}^{*}+\alpha \theta_{0}$ & $-\alpha^{-1}$ & $\theta_{0}+\alpha^{-1} \theta_{d}^{*}$
\end{tabular}

(iii) Assume $\varphi_{1}=\varphi_{d}$ and $\phi_{1} \neq \phi_{d}$. Then $A, A^{*}$ is isomorphic to $\sqrt{40}$ ) if and only if $\xi=1$, $\zeta=0, \xi^{*}=1, \zeta^{*}=0$. Moreover $A^{*}, A$ is isomorphic to 40) if and only if $\xi=-\alpha$, $\zeta=\theta_{d}^{*}+\alpha \theta_{0}, \xi^{*}=-\alpha^{-1}, \zeta^{*}=\theta_{0}+\alpha^{-1} \theta_{d}^{*}$.

(iv) Assume $\phi_{1}=\phi_{d}$ and $\varphi_{1} \neq \varphi_{d}$. Then $A, A^{*}$ is isomorphic to (40) if and only if $\xi=1$, $\zeta=0, \xi^{*}=1, \zeta^{*}=0$. Moreover $A^{*}, A$ is isomorphic to 40 ) if and only if $\xi=\alpha$, $\zeta=\theta_{0}^{*}-\alpha \theta_{0}, \xi^{*}=\alpha^{-1}, \zeta^{*}=\theta_{0}-\alpha^{-1} \theta_{0}^{*}$.

(v) Assume $\varphi_{1}=-\phi_{1}, \varphi_{d}=-\phi_{d}$ and $\varphi_{1} \neq \varphi_{d}$. Then $A, A^{*}$ is isomorphic to 40) if and only if the sequence $\xi, \zeta, \xi^{*}, \zeta^{*}$ is listed in the following table.

\begin{tabular}{cccc}
$\xi$ & $\zeta$ & $\xi^{*}$ & $\zeta^{*}$ \\
\hline 1 & 0 & 1 & 0 \\
-1 & $\theta_{0}+\theta_{d}$ & 1 & 0
\end{tabular}

Moreover $A^{*}, A$ is not isomorphic to 40) for any $\xi, \zeta, \xi^{*}, \zeta^{*}$. 
(vi) Assume $\varphi_{1}=-\phi_{d}, \varphi_{d}=-\phi_{1}$ and $\varphi_{1} \neq \varphi_{d}$. Then $A, A^{*}$ is isomorphic to 40) if and only if the sequence $\xi, \zeta, \xi^{*}, \zeta^{*}$ is listed in the following table.

\begin{tabular}{cccc}
$\xi$ & $\zeta$ & $\xi^{*}$ & $\zeta^{*}$ \\
\hline 1 & 0 & 1 & 0 \\
1 & 0 & -1 & $\theta_{0}^{*}+\theta_{d}^{*}$
\end{tabular}

Moreover $A^{*}, A$ is not isomorphic to (40) for any $\xi, \zeta, \xi^{*}, \zeta^{*}$.

(vii) Assume none of (i)-(vi) hold above. Then $A, A^{*}$ is isomorphic to (40) if and only if $\xi=1, \zeta=0, \xi^{*}=1, \zeta^{*}=0$. Moreover $A^{*}, A$ is not isomorphic to (40) for any $\xi, \zeta, \xi^{*}, \zeta^{*}$.

Proof. Routine consequence of Propositions 11.2 and 11.3 .

\section{The parameter arrrays in closed form}

In [18] and [36] the parameter array of a Leonard system is given in closed form. For the rest of this paper we consider how the results of previous sections look in terms of this form.

Notation 12.1 Let $\Phi=\left(A ;\left\{E_{i}\right\}_{i=0}^{d} ; A^{*} ;\left\{E_{i}^{*}\right\}_{i=0}^{d}\right)$ denote a Leonard system over $\mathbb{K}$ and let $\left(\left\{\theta_{i}\right\}_{i=0}^{d} ;\left\{\theta_{i}^{*}\right\}_{i=0}^{d} ;\left\{\varphi_{i}\right\}_{i=1}^{d} ;\left\{\phi_{i}\right\}_{i=1}^{d}\right)$ denote the corresponding parameter array. We assume $d \geq 3$.

Notation 12.2 Referring to Notation 12.1 let $\overline{\mathbb{K}}$ denote the algebraic closure of $\mathbb{K}$ and let $q$ denote a nonzero scalar in $\overline{\mathbb{K}}$ such that $q+q^{-1}+1$ is equal to the common value of (9). We consider the following types:

\begin{tabular}{c|c} 
type & description \\
\hline I & $q \neq 1, q \neq-1$ \\
II & $q=1$, Char $(\mathbb{K}) \neq 2$ \\
III $^{+}$ & $q=-1$, Char $(\mathbb{K}) \neq 2, d$ even \\
III $^{-}$ & $q=-1$, Char $(\mathbb{K}) \neq 2, d$ odd \\
IV & $q=1$, Char $(\mathbb{K})=2$
\end{tabular}

\section{Type I: $q \neq 1$ and $q \neq-1$}

Lemma 13.1 [18, Theorem 6.1] Referring to Notation 12.1, assume $\Phi$ is Type I. Then there exists unique scalars $\eta, \mu, h, \eta^{*}, \mu^{*}, h^{*}, \tau$ in $\overline{\mathbb{K}}$ such that

$$
\begin{aligned}
& \theta_{i}=\eta+\mu q^{i}+h q^{d-i}, \\
& \theta_{i}^{*}=\eta^{*}+\mu^{*} q^{i}+h^{*} q^{d-i}
\end{aligned}
$$

for $0 \leq i \leq d$ and

$$
\begin{aligned}
\varphi_{i} & =\left(q^{i}-1\right)\left(q^{d-i+1}-1\right)\left(\tau-\mu \mu^{*} q^{i-1}-h h^{*} q^{d-i}\right) \\
\phi_{i} & =\left(q^{i}-1\right)\left(q^{d-i+1}-1\right)\left(\tau-h \mu^{*} q^{i-1}-\mu h^{*} q^{d-i}\right)
\end{aligned}
$$

for $1 \leq i \leq d$ 
Remark 13.2 Referring to Lemma 13.1, for $1 \leq i \leq d$ we have $q^{i} \neq 1$; otherwise $\varphi_{i}=0$ by (43). For $0 \leq i \leq d-1$ we have $\mu \neq h q^{i}$; otherwise $\theta_{d-i}=\theta_{0}$. Similarly $\mu^{*} \neq h^{*} q^{i}$.

Lemma 13.3 Referring to Notation 12.1, assume $\Phi$ is Type I. Then (i)-(iv) hold below.

(i) $\theta_{i}+\theta_{d-i}$ is independent of $i$ for $0 \leq i \leq d$ if and only if $\mu=-h$.

(ii) $\theta_{i}^{*}+\theta_{d-i}^{*}$ is independent of $i$ for $0 \leq i \leq d$ if and only if $\mu^{*}=-h^{*}$.

(iii) $\left(\theta_{i}^{*}-\theta_{0}^{*}\right)\left(\theta_{i}-\theta_{0}\right)^{-1}$ is independent of $i$ for $1 \leq i \leq d$ if and only if $\mu h^{*}=\mu^{*} h$.

(iv) $\left(\theta_{d-i}^{*}-\theta_{d}^{*}\right)\left(\theta_{i}-\theta_{0}\right)^{-1}$ is independent of $i$ for $1 \leq i \leq d$ if and only if $\mu \mu^{*}=h h^{*}$.

Proof. (i): Using (41),

$$
\theta_{i}+\theta_{d-i}-\theta_{0}-\theta_{d}=\left(q^{i}-1\right)\left(1-q^{d-i}\right)(\mu+h)
$$

for $0 \leq i \leq d$. The result follows from this and Remark 13.2.

(ii): Similar to the proof of (i).

(iii): Using (41) and (42),

$$
\frac{\theta_{i}^{*}-\theta_{0}^{*}}{\theta_{i}-\theta_{0}}-\frac{\theta_{d}^{*}-\theta_{0}^{*}}{\theta_{d}-\theta_{0}}=\frac{\left(\mu h^{*}-\mu^{*} h\right)\left(1-q^{d-i}\right)}{(\mu-h)\left(\mu-h q^{d-i}\right)}
$$

for $1 \leq i \leq d$. The result follows from this and Remark 13.2.

(iv): Using (41) and (42),

$$
\frac{\theta_{d-i}^{*}-\theta_{d}^{*}}{\theta_{i}-\theta_{0}}-\frac{\theta_{0}^{*}-\theta_{d}^{*}}{\theta_{d}-\theta_{0}}=\frac{\left(\mu \mu^{*}-h h^{*}\right)\left(1-q^{d-i}\right)}{(\mu-h)\left(\mu-h q^{d-i}\right)}
$$

for $1 \leq i \leq d$. The result follows from this and Remark 13.2.

Lemma 13.4 Referring to Notation 12.1, assume $\Phi$ is Type I. Then (i)-(iv) hold below.

(i) $\varphi_{i}=-\phi_{i}$ for $1 \leq i \leq d$ if and only if $\tau=0$ and $\mu=-h$.

(ii) $\varphi_{i}=-\phi_{d-i+1}$ for $1 \leq i \leq d$ if and only if $\tau=0$ and $\mu^{*}=-h^{*}$.

(iii) $\phi_{i}=\phi_{d-i+1}$ for $1 \leq i \leq d$ if and only if $\mu h^{*}=\mu^{*} h$.

(iv) $\varphi_{i}=\varphi_{d-i+1}$ for $1 \leq i \leq d$ if and only if $\mu \mu^{*}=h h^{*}$.

Proof. (i): Using the data in Lemma 13.1 we find

$$
\varphi_{i}+\phi_{i}=\left(q^{i}-1\right)\left(q^{d-i+1}-1\right)\left(2 \tau-(\mu+h)\left(\mu^{*} q^{i-1}+h^{*} q^{d-i}\right)\right)
$$

for $1 \leq i \leq d$ and

$$
\varphi_{1}+\phi_{1}-\varphi_{d}-\phi_{d}=(q-1)\left(q^{d-1}-1\right)\left(q^{d}-1\right)(\mu+h)\left(\mu^{*}-h^{*}\right) .
$$


First assume $\varphi_{i}=-\phi_{i}$ for $1 \leq i \leq d$. Then in (46) the expression on the left is zero so the expression on the right is zero. In this expression each factor except $\mu+h$ is nonzero by Remark 13.2, so $\mu=-h$. In (45) the expression on the left is zero so the expression on the right is zero. Evaluating this expression using $\mu=-h$ and Remark 13.2 we find $2 \tau=0$. Note that $\operatorname{Char}(\mathbb{K}) \neq 2$; otherwise $\mu=h$ and Remark 13.2 is contradicted. Therefore $\tau=0$. We have now shown $\tau=0$ and $\mu=-h$. Conversely assume $\tau=0$ and $\mu=-h$. Then by (45) we have $\varphi_{i}=-\phi_{i}$ for $1 \leq i \leq d$.

(ii): Similar to the proof of (i). We note that

$$
\varphi_{i}+\phi_{d-i+1}=\left(q^{i}-1\right)\left(q^{d-i+1}-1\right)\left(2 \tau-\left(\mu^{*}+h^{*}\right)\left(\mu q^{i-1}+h q^{d-i}\right)\right)
$$

for $1 \leq i \leq d$ and

$$
\varphi_{1}+\phi_{d}-\varphi_{d}-\phi_{1}=(q-1)\left(q^{d-1}-1\right)\left(q^{d}-1\right)(\mu-h)\left(\mu^{*}+h^{*}\right) .
$$

(iii): Using the data in Lemma 13.1 we find

$$
\phi_{i}-\phi_{d-i+1}=\left(q^{i}-1\right)\left(q^{d-i+1}-1\right)\left(q^{i-1}-q^{d-i}\right)\left(\mu h^{*}-\mu^{*} h\right)
$$

for $1 \leq i \leq d$. The result follows from this and Remark 13.2.

(iv): Using the data in Lemma 13.1 we find

$$
\varphi_{i}-\varphi_{d-i+1}=\left(q^{i}-1\right)\left(q^{d-i+1}-1\right)\left(q^{d-i}-q^{i-1}\right)\left(\mu \mu^{*}-h h^{*}\right)
$$

for $1 \leq i \leq d$. The result follows from this and Remark 13.2 .

Proposition 13.5 Referring to Notation [12.1, assume $\Phi$ is Type I. Then (i)-(vii) hold below.

(i) $\Phi^{\downarrow}$ is affine isomorphic to $\Phi$ if and only if $\mu^{*}=-h^{*}, \tau=0$.

(ii) $\Phi^{\Downarrow}$ is affine isomorphic to $\Phi$ if and only if $\mu=-h, \tau=0$.

(iii) $\Phi^{\downarrow \Downarrow}$ is affine isomorphic to $\Phi$ if and only if $\mu=-h, \mu^{*}=-h^{*}$.

(iv) $\Phi^{*}$ is affine isomorphic to $\Phi$ if and only if $\mu h^{*}=\mu^{*} h$.

(v) $\Phi^{\downarrow *}$ is affine isomorphic to $\Phi$ if and only if $\mu=-h, \mu^{*}=-h^{*}, \tau=0$.

(vi) $\Phi^{\Downarrow *}$ is affine isomorphic to $\Phi$ if and only if $\mu=-h, \mu^{*}=-h^{*}, \tau=0$.

(vii) $\Phi^{\downarrow \Downarrow *}$ is affine isomorphic to $\Phi$ if and only if $\mu \mu^{*}=h h^{*}$.

Proof. Follows from Propositions 8.4, 8.5, 8.7, 8.8, 8.10, 8.12, 8.13, and Lemmas 13.3, 13.4 
Theorem 13.6 Referring to Notation 12.1, assume $\Phi$ is Type I. Then (i)-(vii) hold below.

(i) Case (i) of Theorem 5.4 occurs if and only if $\mu=-h, \mu^{*}=-h^{*}$ and $\tau=0$.

(ii) Case (ii) of Theorem 5.4 occurs if and only if $\mu=-h, \mu^{*}=-h^{*}$ and $\tau \neq 0$.

(iii) Case (iii) of Theorem 5.4 occurs if and only if $\mu \mu^{*}=h h^{*}$ and $\mu h^{*} \neq \mu^{*} h$.

(iv) Case (iv) of Theorem 5.4 occurs if and only if $\mu \mu^{*} \neq h h^{*}$ and $\mu h^{*}=\mu^{*} h$.

(v) Case (v) of Theorem 5.4 occurs if and only if $\mu=-h, \mu^{*} \neq-h^{*}$ and $\tau=0$.

(vi) Case (vi) of Theorem 5.4 occurs if and only if $\mu \neq-h, \mu^{*}=-h^{*}$ and $\tau=0$.

(vii) Case (vii) of Theorem 5.4 occurs if and only if $\mu \mu^{*} \neq h h^{*}, \mu h^{*} \neq \mu^{*} h$, and at least two of $\mu \neq-h, \mu^{*} \neq-h^{*}, \tau \neq 0$.

Proof. Combine Theorem 5.4 and Proposition 13.5 .

\section{Type II: $q=1$ and $\operatorname{Char}(\mathbb{K}) \neq 2$}

Lemma 14.1 [18, Theorem 7.1] Referring to Notation 12.1, assume $\Phi$ is Type II. Then there exists unique scalars $\eta, \mu, h, \eta^{*}, \mu^{*}, h^{*}, \tau$ in $\overline{\mathbb{K}}$ such that

$$
\begin{aligned}
\theta_{i} & =\eta+\mu(i-d / 2)+h i(d-i), \\
\theta_{i}^{*} & =\eta^{*}+\mu^{*}(i-d / 2)+h^{*} i(d-i)
\end{aligned}
$$

for $0 \leq i \leq d$ and

$$
\begin{aligned}
\varphi_{i} & =i(d-i+1)\left(\tau-\mu \mu^{*} / 2+\left(h \mu^{*}+\mu h^{*}\right)(i-(d+1) / 2)+h h^{*}(i-1)(d-i)\right), \\
\phi_{i} & =i(d-i+1)\left(\tau+\mu \mu^{*} / 2+\left(h \mu^{*}-\mu h^{*}\right)(i-(d+1) / 2)+h h^{*}(i-1)(d-i)\right)
\end{aligned}
$$

for $1 \leq i \leq d$.

Remark 14.2 Referring to Lemma 14.1, for $0 \leq i \leq d-1$ we have $\mu \neq-i h$; otherwise $\theta_{d-i}=\theta_{0}$. Similarly $\mu^{*} \neq-i h^{*}$. For any prime $i$ such that $i \leq d$ we have $\operatorname{Char}(\mathbb{K}) \neq i$; otherwise $\varphi_{i}=0$ by (49).

Lemma 14.3 Referring to Notation 12.1, assume $\Phi$ is Type II. Then (i)-(iv) hold below.

(i) $\theta_{i}+\theta_{d-i}$ is independent of $i$ for $0 \leq i \leq d$ if and only if $h=0$.

(ii) $\theta_{i}^{*}+\theta_{d-i}^{*}$ is independent of $i$ for $0 \leq i \leq d$ if and only if $h^{*}=0$.

(iii) $\left(\theta_{i}^{*}-\theta_{0}^{*}\right)\left(\theta_{i}-\theta_{0}\right)^{-1}$ is independent of $i$ for $1 \leq i \leq d$ if and only if $\mu h^{*}=\mu^{*} h$.

(iv) $\left(\theta_{d-i}^{*}-\theta_{d}^{*}\right)\left(\theta_{i}-\theta_{0}\right)^{-1}$ is independent of $i$ for $1 \leq i \leq d$ if and only if $\mu h^{*}=-\mu^{*} h$.

Proof. (i): Using (47),

$$
\theta_{i}+\theta_{d-i}-\theta_{0}-\theta_{d}=2 i(d-i) h
$$


for $0 \leq i \leq d$. The result follows from this and Remark 14.2 .

(ii): Similar to the proof of (i).

(iii): Using (47) and (48),

$$
\frac{\theta_{i}^{*}-\theta_{0}^{*}}{\theta_{i}-\theta_{0}}-\frac{\theta_{d}^{*}-\theta_{0}^{*}}{\theta_{d}-\theta_{0}}=\frac{(d-i)\left(\mu h^{*}-\mu^{*} h\right)}{\mu(\mu+(d-i) h)}
$$

for $1 \leq i \leq d$. The result follows from this and Remark 14.2.

(iv): Using (47) and (48),

$$
\frac{\theta_{d-i}^{*}-\theta_{d}^{*}}{\theta_{i}-\theta_{0}}-\frac{\theta_{0}^{*}-\theta_{d}^{*}}{\theta_{d}-\theta_{0}}=\frac{(d-i)\left(\mu h^{*}+\mu^{*} h\right)}{\mu(\mu+(d-i) h)}
$$

for $1 \leq i \leq d$. The result follows from this and Remark 14.2.

Lemma 14.4 Referring to Notation 12.1, assume $\Phi$ is Type II. Then (i)-(iv) hold below.

(i) $\varphi_{i}=-\phi_{i}$ for $1 \leq i \leq d$ if and only if $\tau=0$ and $h=0$.

(ii) $\varphi_{i}=-\phi_{d-i+1}$ for $1 \leq i \leq d$ if and only if $\tau=0$ and $h^{*}=0$.

(iii) $\phi_{i}=\phi_{d-i+1}$ for $1 \leq i \leq d$ if and only if $\mu h^{*}=\mu^{*} h$.

(iv) $\varphi_{i}=\varphi_{d-i+1}$ for $1 \leq i \leq d$ if and only if $\mu h^{*}=-\mu^{*} h$.

Proof. (i): Using the data in Lemma 14.1 we find

$$
\varphi_{i}+\phi_{i}=2 i(d-i+1)\left(\tau+\mu^{*} h(i-(d+1) / 2)+h h^{*}(i-1)(d-i)\right)
$$

for $1 \leq i \leq d$ and

$$
\varphi_{1}+\phi_{1}-\varphi_{d}-\phi_{d}=2 d(1-d) \mu^{*} h .
$$

First assume $\varphi_{i}=-\phi_{i}$ for $1 \leq i \leq d$. Then in (52) the expression on the left is zero so the expression on the right is zero. In this expression each factor except $h$ is nonzero by Remark 14.2, so $h=0$. In (51) the expression on the left is zero so the expression on the right is zero. Evaluating this expression using $h=0$ and Remark 14.2 we find $\tau=0$. We have now shown $\tau=0$ and $h=0$. Conversely assume $\tau=0$ and $h=0$. Then by (51) we have $\varphi_{i}=-\phi_{i}$ for $1 \leq i \leq d$.

(ii): Similar to the proof of (i). We note that

$$
\varphi_{i}+\phi_{d-i+1}=2 i(d-i+1)\left(\tau+\mu h^{*}(i-(d+1) / 2)+h h^{*}(i-1)(d-i)\right)
$$

for $1 \leq i \leq d$ and

$$
\varphi_{1}+\phi_{d}-\varphi_{d}-\phi_{1}=2 d(1-d) \mu h^{*} .
$$

(iii): Using the data in Lemma 14.1 we find

$$
\phi_{i}-\phi_{d-i+1}=i(d-i+1)(d-2 i+1)\left(\mu h^{*}-\mu^{*} h\right)
$$

for $1 \leq i \leq d$. The result follows from this and Remark 14.2.

(iv): Using the data in Lemma 14.1 we find

$$
\varphi_{i}-\varphi_{d-i+1}=-i(d-i+1)(d-2 i+1)\left(\mu h^{*}+\mu^{*} h\right)
$$

for $1 \leq i \leq d$. The result follows from this and Remark 14.2. 
Proposition 14.5 Referring to Notation 12.1, assume $\Phi$ is Type II. Then (i)-(vii) hold below.

(i) $\Phi^{\downarrow}$ is affine isomorphic to $\Phi$ if and only if $h^{*}=0, \tau=0$.

(ii) $\Phi^{\Downarrow}$ is affine isomorphic to $\Phi$ if and only if $h=0, \tau=0$.

(iii) $\Phi^{\downarrow \Downarrow}$ is affine isomorphic to $\Phi$ if and only if $h=0, h^{*}=0$.

(iv) $\Phi^{*}$ is affine isomorphic to $\Phi$ if and only if $\mu h^{*}=\mu^{*} h$.

(v) $\Phi^{\downarrow *}$ is affine isomorphic to $\Phi$ if and only if $h=0, h^{*}=0, \tau=0$.

(vi) $\Phi^{\Downarrow *}$ is affine isomorphic to $\Phi$ if and only if $h=0, h^{*}=0, \tau=0$.

(vii) $\Phi^{\downarrow \Downarrow *}$ is affine isomorphic to $\Phi$ if and only if $\mu h^{*}=-\mu^{*} h$.

Proof. Follows from Propositions 8.4, 8.5, 8.7, 8.8, 8.10, 8.12, 8.13, and Lemmas 14.3, 14.4 .

Theorem 14.6 Referring to Notation 12.1, assume $\Phi$ is Type II. Then (i)-(vii) hold below.

(i) Case (i) of Theorem 5.4 occurs if and only if $h=0, h^{*}=0$ and $\tau=0$.

(ii) Case (ii) of Theorem 5.4 occurs if and only if $h=0, h^{*}=0$ and $\tau \neq 0$.

(iii) Case (iii) of Theorem 5.4 occurs if and only if $\mu h^{*} \neq \mu^{*} h$ and $\mu h^{*}=-\mu^{*} h$.

(iv) Case (iv) of Theorem 5.4 occurs if and only if $\mu h^{*}=\mu^{*} h$ and $\mu h^{*} \neq-\mu^{*} h$.

(v) Case (v) of Theorem 5.4 occurs if and only if $h=0, h^{*} \neq 0$ and $\tau=0$.

(vi) Case (vi) of Theorem 5.4 occurs if and only if $h \neq 0, h^{*}=0$ and $\tau=0$.

(vii) Case (vii) of Theorem 5.4 occurs if and only if $\mu h^{*} \neq \mu^{*} h, \mu h^{*} \neq-\mu^{*} h$, and at least two of $h, h^{*}, \tau$ are nonzero.

Proof. Combine Theorem 5.4 and Proposition 14.5 . 


\section{Type III $^{+}: q=-1, \operatorname{Char}(\mathbb{K}) \neq 2$ and $d$ is even}

Lemma 15.1 [18, Theorem 8.1] Referring to Notation 12.1, assume $\Phi$ is Type $I I I^{+}$. Then there exists unique scalars $\eta, h, s, \eta^{*}, h^{*}, s^{*}, \tau$ in $\overline{\mathbb{K}}$ such that

$$
\begin{aligned}
\theta_{i} & = \begin{cases}\eta+s+h(i-d / 2) & \text { if } i \text { is even, } \\
\eta-s-h(i-d / 2) & \text { if } i \text { is odd, }\end{cases} \\
\theta_{i}^{*} & = \begin{cases}\eta^{*}+s^{*}+h^{*}(i-d / 2) & \text { if } i \text { is even, } \\
\eta^{*}-s^{*}-h^{*}(i-d / 2) & \text { if } i \text { is odd }\end{cases}
\end{aligned}
$$

for $0 \leq i \leq d$ and

$$
\begin{aligned}
\varphi_{i} & = \begin{cases}i\left(\tau-s h^{*}-s^{*} h-h h^{*}(i-(d+1) / 2)\right) & \text { if } i \text { is even, } \\
(d-i+1)\left(\tau+s h^{*}+s^{*} h+h h^{*}(i-(d+1) / 2)\right) & \text { if } i \text { is odd, }\end{cases} \\
\phi_{i} & = \begin{cases}i\left(\tau-s h^{*}+s^{*} h+h h^{*}(i-(d+1) / 2)\right) & \text { if } i \text { is even, } \\
(d-i+1)\left(\tau+s h^{*}-s^{*} h-h h^{*}(i-(d+1) / 2)\right) & \text { if } i \text { is odd }\end{cases}
\end{aligned}
$$

for $1 \leq i \leq d$.

Remark 15.2 Referring to Lemma 15.1, we have $h \neq 0$; otherwise $\theta_{0}=\theta_{2}$ by (53). Similary we have $h^{*} \neq 0$. For $i$ odd with $0 \leq i \leq d-1$ we have $s \neq i h / 2$; otherwise $\theta_{d-i}=\theta_{0}$. For any prime $i$ such that $i \leq d / 2$ we have $\operatorname{Char}(\mathbb{K}) \neq i$; otherwise $\varphi_{2 i}=0$ by (55). By this and since $\operatorname{Char}(\mathbb{K}) \neq 2$ we find $\operatorname{Char}(\mathbb{K})$ is either 0 or an odd prime greater than $d / 2$. Observe neither of $d, d-2$ vanish in $\mathbb{K}$ since otherwise Char $(\mathbb{K})$ must divide $d / 2$ or $(d-2) / 2$.

Lemma 15.3 Referring to Notation 12.1, assume $\Phi$ is Type $I I I^{+}$. Then (i)-(iv) hold below.

(i) $\theta_{i}+\theta_{d-i}$ is independent of $i$ for $0 \leq i \leq d$ if and only if $s=0$.

(ii) $\theta_{i}^{*}+\theta_{d-i}^{*}$ is independent of $i$ for $0 \leq i \leq d$ if and only if $s^{*}=0$.

(iii) $\left(\theta_{i}^{*}-\theta_{0}^{*}\right)\left(\theta_{i}-\theta_{0}\right)^{-1}$ is independent of $i$ for $1 \leq i \leq d$ if and only if $h s^{*}=h^{*} s$.

(iv) $\left(\theta_{d-i}^{*}-\theta_{d}^{*}\right)\left(\theta_{i}-\theta_{0}\right)^{-1}$ is independent of $i$ for $1 \leq i \leq d$ if and only if $h s^{*}=-h^{*} s$.

Proof. (i): Using (53),

$$
\theta_{i}+\theta_{d-i}= \begin{cases}2(\eta+s) & \text { if } i \text { is even } \\ 2(\eta-s) & \text { if } i \text { is odd }\end{cases}
$$

for $0 \leq i \leq d$. The result follows from this.

(ii): Similar to the proof of (i).

(iii): Using (53) and (54),

$$
\frac{\theta_{i}^{*}-\theta_{0}^{*}}{\theta_{i}-\theta_{0}}-\frac{\theta_{d}^{*}-\theta_{0}^{*}}{\theta_{d}-\theta_{0}}= \begin{cases}0 & \text { if } i \text { is even } \\ \frac{h s^{*}-h^{*} s}{h(s-h(d-i) / 2)} & \text { if } i \text { is odd }\end{cases}
$$


for $1 \leq i \leq d$. The result follows from this.

(iv): Using (53) and (54),

$$
\frac{\theta_{d-i}^{*}-\theta_{d}^{*}}{\theta_{i}-\theta_{0}}-\frac{\theta_{0}^{*}-\theta_{d}^{*}}{\theta_{d}-\theta_{0}}= \begin{cases}0 & \text { if } i \text { is even } \\ \frac{h s^{*}+h^{*} s}{h(s-h(d-i) / 2)} & \text { if } i \text { is odd }\end{cases}
$$

for $1 \leq i \leq d$. The result follows from this.

Lemma 15.4 Referring to Notation 12.1, assume $\Phi$ is Type $I I I^{+}$. Then (i)-(iv) hold below.

(i) $\varphi_{i}=-\phi_{i}$ for $1 \leq i \leq d$ if and only if $\tau=0$ and $s=0$.

(ii) $\varphi_{i}=-\phi_{d-i+1}$ for $1 \leq i \leq d$ if and only if $\tau=0$ and $s^{*}=0$.

(iii) $\phi_{i}=\phi_{d-i+1}$ for $1 \leq i \leq d$ if and only if $h s^{*}=h^{*} s$.

(iv) $\varphi_{i}=\varphi_{d-i+1}$ for $1 \leq i \leq d$ if and only if $h s^{*}=-h^{*} s$.

Proof. (i): Using the data in Lemma 15.1 we find

$$
\varphi_{i}+\phi_{i}= \begin{cases}2 i\left(\tau-h^{*} s\right) & \text { if } i \text { is even } \\ 2(d-i+1)\left(\tau+h^{*} s\right) & \text { if } i \text { is odd }\end{cases}
$$

for $1 \leq i \leq d$. The result follows from this and Remark 15.2,

(ii): Using the data in Lemma 15.1 we find

$$
\varphi_{i}+\phi_{d-i+1}= \begin{cases}2 i\left(\tau-h s^{*}\right) & \text { if } i \text { is even, } \\ 2(d-i+1)\left(\tau+h s^{*}\right) & \text { if } i \text { is odd }\end{cases}
$$

for $1 \leq i \leq d$. The result follows from this and Remark 15.2.

(iii): Using the data in Lemma 15.1 we find

$$
\phi_{i}-\phi_{d-i+1}= \begin{cases}2 i\left(h s^{*}-h^{*} s\right) & \text { if } i \text { is even } \\ -2(d-i+1)\left(h s^{*}-h^{*} s\right) & \text { if } i \text { is odd }\end{cases}
$$

for $1 \leq i \leq d$. The result follows from this and Remark 15.2,

(iv): Using the data in Lemma 15.1 we find

$$
\varphi_{i}-\varphi_{d-i+1}= \begin{cases}-2 i\left(h s^{*}+h^{*} s\right) & \text { if } i \text { is even, } \\ 2(d-i+1)\left(h s^{*}+h^{*} s\right) & \text { if } i \text { is odd }\end{cases}
$$

for $1 \leq i \leq d$. The result follows from this and Remark 15.2 . 
Proposition 15.5 Referring to Notation 12.1, assume $\Phi$ is Type III ${ }^{+}$. Then (i)-(vii) hold below.

(i) $\Phi^{\downarrow}$ is affine isomorphic to $\Phi$ if and only if $s^{*}=0, \tau=0$.

(ii) $\Phi^{\Downarrow}$ is affine isomorphic to $\Phi$ if and only if $s=0, \tau=0$.

(iii) $\Phi^{\downarrow \Downarrow}$ is affine isomorphic to $\Phi$ if and only if $s=0, s^{*}=0$.

(iv) $\Phi^{*}$ is affine isomorphic to $\Phi$ if and only if $h s^{*}=h^{*} s$.

(v) $\Phi^{\downarrow *}$ is affine isomorphic to $\Phi$ if and only if $s=0, s^{*}=0, \tau=0$.

(vi) $\Phi^{\Downarrow *}$ is affine isomorphic to $\Phi$ if and only if $s=0, s^{*}=0, \tau=0$.

(vii) $\Phi^{\downarrow \Downarrow *}$ is affine isomorphic to $\Phi$ if and only if $h s^{*}=-h^{*} s$.

Proof. Follows from Propositions 8.4, 8.5, 8.7, 8.8, 8.10, 8.12, 8.13, and Lemmas 15.3, 15.4 .

Theorem 15.6 Referring to Notation 12.1, assume $\Phi$ is Type $I I I^{+}$. Then (i)-(vii) hold below.

(i) Case (i) of Theorem 5.4 occurs if and only if $s=0, s^{*}=0$ and $\tau=0$.

(ii) Case (ii) of Theorem 5.4 occurs if and only if $s=0, s^{*}=0$ and $\tau \neq 0$.

(iii) Case (iii) of Theorem 5.4 occurs if and only if $h s^{*} \neq h^{*} s$ and $h s^{*}=-h^{*} s$.

(iv) Case (iv) of Theorem 5.4 occurs if and only if $h s^{*}=h^{*} s$ and $h s^{*} \neq-h^{*} s$.

(v) Case (v) of Theorem 5.4 occurs if and only if $s=0, s^{*} \neq 0$ and $\tau=0$.

(vi) Case (vi) of Theorem 5.4 occurs if and only if $s \neq 0, s^{*}=0$ and $\tau=0$.

(vii) Case (vii) of Theorem 5.4 occurs if and only if $h s^{*} \neq h^{*} s, h s^{*} \neq-h^{*} s$, and at least two of $s, s^{*}, \tau$ are nonzero.

Proof. Combine Theorem 5.4 and Proposition 15.5 . 


\section{Type III $^{-}: q=-1, \operatorname{Char}(\mathbb{K}) \neq 2$ and $d$ is odd}

Lemma 16.1 [18, Theorem 9.1] Referring to Notation 12.1, assume $\Phi$ is Type III- . Then there exists unique scalars $\eta, h, s, \eta^{*}, h^{*}, s^{*}, \tau$ in $\overline{\mathbb{K}}$ such that

$$
\begin{aligned}
\theta_{i} & = \begin{cases}\eta+s+h(i-d / 2) & \text { if } i \text { is even, } \\
\eta-s-h(i-d / 2) & \text { if } i \text { is odd, }\end{cases} \\
\theta_{i}^{*} & = \begin{cases}\eta^{*}+s^{*}+h^{*}(i-d / 2) & \text { if } i \text { is even, } \\
\eta^{*}-s^{*}-h^{*}(i-d / 2) & \text { if } i \text { is odd }\end{cases}
\end{aligned}
$$

for $0 \leq i \leq d$ and

$$
\begin{aligned}
\varphi_{i} & = \begin{cases}h h^{*} i(d-i+1) & \text { if } i \text { is even, } \\
\tau-2 s s^{*}+i(d-i+1) h h^{*}-2\left(h s^{*}+h^{*} s\right)(i-(d+1) / 2) & \text { if } i \text { is odd, }\end{cases} \\
\phi_{i} & = \begin{cases}h h^{*} i(d-i+1) & \text { if } i \text { is even, } \\
\tau+2 s s^{*}+i(d-i+1) h h^{*}-2\left(h s^{*}-h^{*} s\right)(i-(d+1) / 2) & \text { if } i \text { is odd }\end{cases}
\end{aligned}
$$

for $1 \leq i \leq d$.

Remark 16.2 Referring to Lemma [16.1, we have $h \neq 0$; otherwise $\theta_{0}=\theta_{2}$ by (57). Similary we have $h^{*} \neq 0$. We have $s \neq 0$; otherwise $\theta_{0}=\theta_{d}$ by (57). Similarly we have $s^{*} \neq 0$. For $i$ even with $0 \leq i \leq d-1$ we have $s \neq i h / 2$; otherwise $\theta_{d-i}=\theta_{0}$. For any prime $i$ such that $i \leq d / 2$ we have $\operatorname{Char}(\mathbb{K}) \neq i$; otherwise $\varphi_{2 i}=0$ by (59). By this and since $\operatorname{Char}(\mathbb{K}) \neq 2$ we find $\operatorname{Char}(\mathbb{K})$ is either 0 or an odd prime greater than $d / 2$. Observe $d-1$ does not vanish in $\mathbb{K}$ since otherwise $\operatorname{Char}(\mathbb{K})$ must divide $(d-1) / 2$.

Lemma 16.3 Referring to Notation 12.1, assume $\Phi$ is Type $I I I^{-}$. Then (i)-(iv) hold below.

(i) $\theta_{0}+\theta_{d} \neq \theta_{1}+\theta_{d-1}$.

(ii) $\theta_{0}^{*}+\theta_{d}^{*} \neq \theta_{1}^{*}+\theta_{d-1}^{*}$.

(iii) $\left(\theta_{i}^{*}-\theta_{0}^{*}\right)\left(\theta_{i}-\theta_{0}\right)^{-1}$ is independent of $i$ for $1 \leq i \leq d$ if and only if $h s^{*}=h^{*} s$.

(iv) $\left(\theta_{d-i}^{*}-\theta_{d}^{*}\right)\left(\theta_{i}-\theta_{0}\right)^{-1}$ is independent of $i$ for $1 \leq i \leq d$ if and only if $h s^{*}=-h^{*} s$.

Proof. (i): Using (57),

$$
\theta_{0}+\theta_{d}-\theta_{1}-\theta_{d-1}=-2(d-1) h
$$

The result follows from this and Remark 16.2.

(ii): Similar to the proof of (i).

(iii): Using (57) and (58),

$$
\frac{\theta_{i}^{*}-\theta_{0}^{*}}{\theta_{i}-\theta_{0}}-\frac{\theta_{d}^{*}-\theta_{0}^{*}}{\theta_{d}-\theta_{0}}= \begin{cases}\frac{h^{*} s-h s^{*}}{h s} & \text { if } i \text { is even, } \\ \frac{(d-i)\left(h s^{*}-h^{*} s\right)}{s(2 s-h(d-i))} & \text { if } i \text { is odd }\end{cases}
$$


for $1 \leq i \leq d$. The result follows from this.

(iv): Using (57) and (58),

$$
\frac{\theta_{d-i}^{*}-\theta_{d}^{*}}{\theta_{i}-\theta_{0}}-\frac{\theta_{0}^{*}-\theta_{d}^{*}}{\theta_{d}-\theta_{0}}= \begin{cases}\frac{h s^{*}+h^{*} s}{h s} & \text { if } i \text { is even } \\ \frac{(d-i)\left(h s^{*}+h^{*} s\right)}{s(-2 s+h(d-i))} & \text { if } i \text { is odd }\end{cases}
$$

for $1 \leq i \leq d$. The result follows from this.

Lemma 16.4 Referring to Notation 12.1, assume $\Phi$ is Type $I I I^{-}$. Then (i)-(iv) hold below.

(i) $\varphi_{2} \neq-\phi_{2}$. Moreover if $\varphi_{1}=-\phi_{1}$ then $\varphi_{d} \neq-\phi_{d}$.

(ii) $\varphi_{2} \neq-\phi_{d-1}$. Moreover if $\varphi_{1}=-\phi_{d}$ then $\varphi_{d} \neq-\phi_{1}$.

(iii) $\phi_{i}=\phi_{d-i+1}$ for $1 \leq i \leq d$ if and only if $h s^{*}=h^{*} s$.

(iv) $\varphi_{i}=\varphi_{d-i+1}$ for $1 \leq i \leq d$ if and only if $h s^{*}=-h^{*} s$.

Proof. (i): Using the data in Lemma 16.1 we find

$$
\begin{gathered}
\varphi_{2}+\phi_{2}=4(d-1) h h^{*}, \\
\varphi_{1}+\phi_{1}-\varphi_{d}-\phi_{d}=4(d-1) h s^{*} .
\end{gathered}
$$

The result follows from this and Remark 16.2.

(ii): Using the data in Lemma 16.1 we find

$$
\begin{gathered}
\varphi_{2}+\phi_{d-1}=4(d-1) h h^{*}, \\
\varphi_{1}+\phi_{d}-\varphi_{d}-\phi_{1}=4(d-1) h^{*} s .
\end{gathered}
$$

The result follows from this and Remark 16.2,

(iii): Using the data in Lemma 16.1 we find

$$
\phi_{i}-\phi_{d-i+1}= \begin{cases}0 & \text { if } i \text { is even } \\ 2(d-2 i+1)\left(h s^{*}-h^{*} s\right) & \text { if } i \text { is odd }\end{cases}
$$

for $1 \leq i \leq d$. The result follows from this and Remark 16.2.

(iv): Using the data in Lemma 16.1 we find

$$
\varphi_{i}-\varphi_{d-i+1}= \begin{cases}0 & \text { if } i \text { is even } \\ 2(d-2 i+1)\left(h s^{*}+h^{*} s\right) & \text { if } i \text { is odd }\end{cases}
$$

for $1 \leq i \leq d$. The result follows from this and Remark 16.2 . 
Proposition 16.5 Referring to Notation 12.1, assume $\Phi$ is Type III- ${ }^{-}$Then (i)-(vii) hold below.

(i) $\Phi^{\downarrow}$ is not affine isomorphic to $\Phi$.

(ii) $\Phi^{\Downarrow}$ is not affine isomorphic to $\Phi$.

(iii) $\Phi^{\downarrow \Downarrow}$ is not affine isomorphic to $\Phi$.

(iv) $\Phi^{*}$ is affine isomorphic to $\Phi$ if and only if $h s^{*}=h^{*} s$.

(v) $\Phi^{\downarrow *}$ is not affine isomorphic to $\Phi$.

(vi) $\Phi^{\Downarrow *}$ is not affine isomorphic to $\Phi$.

(vii) $\Phi^{\downarrow \Downarrow *}$ is affine isomorphic to $\Phi$ if and only if $h s^{*}=-h^{*} s$.

Proof. Follows from Propositions 8.4, 8.5, 8.7, 8.8, 8.10, 8.12, 8.13, and Lemmas 16.3, 16.4 .

Theorem 16.6 Referring to Notation 12.1, assume $\Phi$ is Type III- . Then (i)-(iv) hold below.

(i) Case (iii) of Theorem 5.4 occurs if and only if $h s^{*}=-h^{*} s$.

(ii) Case (iv) of Theorem 5.4 occurs if and only if $h s^{*}=h^{*} s$.

(iii) Case (vii) of Theorem 5.4 occurs if and only if both $h s^{*} \neq h^{*} s, h s^{*} \neq-h^{*} s$.

(iv) Cases (i), (ii), (v), (vi) of Theorem5.4 do not occur.

Proof. Combine Theorem 5.4 and Proposition 16.5 .

\section{Type IV: $q=1$ and $\operatorname{Char}(\mathbb{K})=2$}

Lemma 17.1 [18, Theorem 10.1] Referring to Notation[12.1, assume $\Phi$ is Type IV. Then $d=3$. Moreover there exists unique scalars $h, s, h^{*}, s^{*}, r$ in $\overline{\mathbb{K}}$ such that

$$
\begin{array}{lll}
\theta_{1}=\theta_{0}+h(s+1), & \theta_{2}=\theta_{0}+h, & \theta_{3}=\theta_{0}+h s, \\
\theta_{1}^{*}=\theta_{0}^{*}+h^{*}\left(s^{*}+1\right), & \theta_{2}^{*}=\theta_{0}^{*}+h^{*}, & \theta_{3}^{*}=\theta_{0}^{*}+h^{*} s^{*}, \\
\varphi_{1}=h h^{*} r, & \varphi_{2}=h h^{*}, & \varphi_{3}=h h^{*}\left(r+s+s^{*}\right), \\
\phi_{1}=h h^{*}\left(r+s\left(1+s^{*}\right)\right), & \phi_{2}=h h^{*}, & \phi_{3}=h h^{*}\left(r+s^{*}(1+s)\right) .
\end{array}
$$

Remark 17.2 Referring to Lemma 17.1, each of $h, h^{*}, s, s^{*}$ is nonzero, and each of $s, s^{*}$ is not equal to 1 . 
Lemma 17.3 Referring to Notation 12.1, assume $\Phi$ is Type IV. Then (i)-(iv) hold below.

(i) $\theta_{i}+\theta_{d-i}=h s$ for $0 \leq i \leq d$.

(ii) $\theta_{i}^{*}+\theta_{d-i}^{*}=h^{*} s^{*}$ for $0 \leq i \leq d$.

(iii) $\left(\theta_{i}^{*}-\theta_{0}^{*}\right)\left(\theta_{i}-\theta_{0}\right)^{-1}$ is independent of $i$ for $1 \leq i \leq d$ if and only if $s=s^{*}$.

(iv) $\left(\theta_{d-i}^{*}-\theta_{d}^{*}\right)\left(\theta_{i}-\theta_{0}\right)^{-1}$ is independent of $i$ for $1 \leq i \leq d$ if and only if $s=s^{*}$.

Proof. (i), (ii): Routine verification using the data in Lemma 17.1.

(iii): Using the data in Lemma 17.1 we find

$$
\frac{\theta_{1}^{*}-\theta_{0}^{*}}{\theta_{1}-\theta_{0}}=\frac{h^{*}\left(s^{*}+1\right)}{h(s+1)}, \quad \frac{\theta_{2}^{*}-\theta_{0}^{*}}{\theta_{2}-\theta_{0}}=\frac{h^{*}}{h}, \quad \frac{\theta_{3}^{*}-\theta_{0}^{*}}{\theta_{3}-\theta_{0}}=\frac{h^{*} s^{*}}{h s} .
$$

The result follows from this and Remark 17.2.

(iv): Using the data in Lemma 17.1 we find

$$
\frac{\theta_{2}^{*}-\theta_{3}^{*}}{\theta_{1}-\theta_{0}}=\frac{h^{*}\left(s^{*}+1\right)}{h(s+1)}, \quad \frac{\theta_{1}^{*}-\theta_{3}^{*}}{\theta_{2}-\theta_{0}}=\frac{h^{*}}{h}, \quad \frac{\theta_{0}^{*}-\theta_{3}^{*}}{\theta_{3}-\theta_{0}}=\frac{h^{*} s^{*}}{h s} .
$$

The result follows from this and Remark 17.2 .

Lemma 17.4 Referring to Notation 12.1, assume $\Phi$ is Type IV. Then (i)-(iv) hold below.

(i) $\varphi_{1} \neq-\phi_{1}$.

(ii) $\varphi_{1} \neq-\phi_{3}$.

(iii) $\phi_{1}=\phi_{3}$ if and only if $s=s^{*}$.

(iv) $\varphi_{1}=\varphi_{3}$ if and only if $s=s^{*}$.

Proof. Using the data in Lemma 17.1 we find

$$
\begin{aligned}
\varphi_{1}+\phi_{1} & =h h^{*} s\left(s^{*}+1\right), & \varphi_{1}+\phi_{3} & =h h^{*} s^{*}(s+1), \\
\phi_{1}-\phi_{3} & =h h^{*}\left(s+s^{*}\right), & \varphi_{1}-\varphi_{3} & =h h^{*}\left(s+s^{*}\right) .
\end{aligned}
$$

Now (i)-(iv) follow from this and Remark 17.2 . 
Proposition 17.5 Referring to Notation 12.1, assume $\Phi$ is Type IV. Then (i)-(vii) hold below.

(i) $\Phi^{\downarrow}$ is not affine isomorphic to $\Phi$.

(ii) $\Phi^{\Downarrow}$ is not affine isomorphic to $\Phi$.

(iii) $\Phi^{\downarrow \Downarrow}$ is affine isomorphic to $\Phi$ if and only if $s=s^{*}$.

(iv) $\Phi^{*}$ is affine isomorphic to $\Phi$ if and only if $s=s^{*}$.

(v) $\Phi^{\downarrow *}$ is not affine isomorphic to $\Phi$.

(vi) $\Phi^{\downarrow *}$ is not affine isomorphic to $\Phi$.

(vii) $\Phi^{\downarrow \Downarrow *}$ is affine isomorphic to $\Phi$ if and only if $s=s^{*}$.

Proof. Follows from Propositions 8.4, 8.5, 8.7, 8.8, 8.10, 8.12, 8.13, and Lemmas 17.3, 17.4

Theorem 17.6 Referring to Notation 12.1, assume $\Phi$ is Type IV. Then (i)-(iii) hold below.

(i) Case (ii) of Theorem 5.4 occurs if and only if $s=s^{*}$.

(ii) Case (vii) of Theorem 5.4 occurs if and only if $s \neq s^{*}$.

(iii) Cases (i), (iii)-(vi) of Theorem5.4 do not occur.

Proof. Combine Theorem 5.4 and Proposition 17.5 .

\section{References}

[1] H. Alnajjar, B. Curtin, A family of tridiagonal pairs, Linear Algebra Appl. 390 (2004) 369-384.

[2] H. Alnajjar, B. Curtin, A family of tridiagonal pairs related to the quantum affine algebra $U_{q}\left(\widehat{\mathfrak{s l}}_{2}\right)$, Electron. J. Linear Algebra 13 (2005) 1-9.

[3] G. Benkart, P. Terwilliger, Irreducible modules for the quantum affine algebra $U_{q}\left(\widehat{\mathfrak{s l}}_{2}\right)$ and its Borel subalgebra, J. Algebra 282 (2004) 172-194; arXiv:math.QA/0311152.

[4] G. Benkart, P. Terwilliger, The universal central extension of the three-point $\mathfrak{s l}_{2}$ loop algebra, Proc. AMS, in press; arXiv:math.RA/0512422.

[5] B. Hartwig, Three mutually adjacent Leonard pairs, Linear Algebra Appl. 408 (2005) 19-39; arXiv:math.AC/0508415. 
[6] B. Hartwig, The Tetrahedron algebra and its finite dimensional irreducible modules, Linear Algebra Appl., in press; arXiv:math.RT/0606197.

[7] B. Hartwig, P. Terwilliger, The tetrahedron algebra, the Onsager algebra, and the $\mathfrak{s l}_{2}$ loop algebra, J. Algebra, in press; arXiv:math-ph/0511004.

[8] T. Ito, K. Tanabe, P. Terwilliger, Some algebra related to $P$ - and $Q$-polynomial association schemes, Codes and Association Schemes (Piscataway NJ, 1999), American Mathematical Society, Providence, RI, 2001, pp. 167-192; arXiv:math.C0/0406556.

[9] T. Ito, P. Terwilliger, The shape of a tridiagonal pair, J. Pure Appl. Algebra 188 (2004) 145-160; arXiv:math.QA/0304244.

[10] T. Ito, P. Terwilliger, Tridiagonal pairs and the quantum affine algebra $U_{q}\left(\widehat{\mathfrak{s l}}_{2}\right)$, Ramanujan J., in press; arXiv:math.QA/0310042.

[11] T. Ito, P. Terwilliger, Two non-nilpotent linear transformations that satisfy the cubic $q$-Serre relations, J. Algebra Appl., in press; arXiv:math.QA/0508398.

[12] T. Ito, P. Terwilliger, The $q$-tetrahedron algebra and its finite dimensional irreducible modules, Comm. Algebra, in press; arXiv:math.QA/0602199.

[13] T. Ito, P. Terwilliger, $q$-inverting pairs of linear transformations and the $q$-tetrahedron algebra, Linear Algebra Appl., submitted for publication; arXiv:math.RT/0606237.

[14] T. Ito, P. Terwilliger, C. Weng, The quantum algebra $U_{q}\left(\mathfrak{s l}_{2}\right)$ and its equitable presentation, J. Algebra 298 (2006) 284-301; arXiv:math.QA/0507477.

[15] K. Nomura, Tridiagonal pairs and the Askey-Wilson relations, Linear Algebra Appl. 397 (2005) 99-106.

[16] K. Nomura, A refinement of the split decomposition of a tridiagonal pair, Linear Algebra Appl. 403 (2005) 1-23.

[17] K. Nomura, Tridiagonal pairs of height one, Linear Algebra Appl. 403 (2005) 118-142.

[18] K. Nomura, P. Terwilliger, Balanced Leonard pairs, Linear Algebra Appl., in press; arXiv:math.RA/0506219.

[19] K. Nomura, P. Terwilliger, Some trace formulae involving the split sequences of a Leonard pair, Linear Algebra Appl. 413 (2006) 189-201; arXiv:math.RA/0508407.

[20] K. Nomura, P. Terwilliger, The determinant of $A A^{*}-A^{*} A$ for a Leonard pair $A, A^{*}$, Linear Algebra Appl. 416 (2006) 880-889; arXiv:math.RA/0511641.

[21] K. Nomura, P. Terwilliger, Matrix units associated with the split basis of a Leonard pair, Linear Algebra Appl. 418 (2006) 775-787; arXiv:math.RA/0602416.

[22] K. Nomura, P. Terwilliger, Linear transformations that are tridiagonal with respect to both eigenbases of a Leonard pair, Linear Algebra Appl., in press; arXiv:math.RA/0605316. 
[23] K. Nomura, P. Terwilliger, The switching element for a Leonard pair, Linear Algebra Appl., submitted for publication; arXiv:math.RA/0608623.

[24] A. A. Pascasio, On the multiplicities of the primitive idempotents of a $Q$-polynomial distance-regular graph, European J. Combin. 23 (2002) 1073-1078.

[25] H. Rosengren, Multivariable orthogonal polynomials and coupling coefficients for discrete series representations, SIAM J. Math. Anal. 30 (1999) 233-272.

[26] H. Rosengren, An elementary approach to the $6 j$-symbols (classical, quantum, rational, trigonometric, and elliptic), Ramanujan J., in press; arXiv:math.CA/0312310.

[27] P. Terwilliger, The subconstituent algebra of an association scheme I, J. Algebraic Combin. 1 (1992) 363-388.

[28] P. Terwilliger, The subconstituent algebra of an association scheme III, J. Algebraic Combin. 2 (1993) 177-210.

[29] P. Terwilliger, Two linear transformations each tridiagonal with respect to an eigenbasis of the other, Linear Algebra Appl. 330 (2001) 149-203; arXiv:math.RA/0406555.

[30] P. Terwilliger, Two relations that generalize the $q$-Serre relations and the Dolan-Grady relations, Physics and Combinatorics 1999 (Nagoya), World Scientific Publishing, River Edge, NJ, 2001, pp. 377-398; arXiv:math.QA/0307016.

[31] P. Terwilliger, Leonard pairs from 24 points of view, Rocky Mountain J. Math. 32 (2) (2002) 827-888; arXiv:math.RA/0406577.

[32] P. Terwilliger, Two linear transformations each tridiagonal with respect to an eigenbasis of the other; the $T D-D$ and the $L B-U B$ canonical form, J. Algebra 291 (2005) $1-45$; arXiv:math.RA/0304077.

[33] P. Terwilliger, Introduction to Leonard pairs, J. Comput. Appl. Math. 153 (2) (2003) 463-475.

[34] P. Terwilliger, Introduction to Leonard pairs and Leonard systems, Sūrikaisekikenkyūsho Kōkyūroku 1109 (1999) 67-79, Algebraic Combinatorics (Kyoto, 1999).

[35] P. Terwilliger, Two linear transformations each tridiagonal with respect to an eigenbasis of the other; comments on the split decomposition, J. Comput. Appl. Math. 178 (2005) 437-452; arXiv:math.RA/0306290.

[36] P. Terwilliger, Two linear transformations each tridiagonal with respect to an eigenbasis of the other; comments on the parameter array, Des. Codes Cryptogr. 34 (2005) 307-332; arXiv:math.RA/0306291.

[37] P. Terwilliger, Leonard pairs and the $q$-Racah polynomials, Linear Algebra Appl. 387 (2004) 235-276; arXiv:math.QA/0306301. 
[38] P. Terwilliger, An algebraic approach to the Askey scheme of orthogonal polynomials, in: Orthogonal Polynomials and Special Functions: computation and applications, Lecture Notes in Mathematics, vol. 1883, Springer, 2006, pp. 255-330; arXiv:math.QA/0408390.

[39] P. Terwilliger, The equitable presentation for the quantum group $U_{q}(\mathfrak{g})$ associated with a symmetrizable Kac-Moody algebra g, J. Algebra 298 (2006) 302-319; arXiv:math.QA/0507478.

[40] P. Terwilliger, R. Vidunas, Leonard pairs and the Askey-Wilson relations, J. Algebra Appl. 3 (2004) 411-426; arXiv:math.QA/0305356.

[41] R. Vidunas, Normalized Leonard pairs and Askey-Wilson relations, Technical Report MHF 2005-16, Kyushu University 2005; arXiv:math.RA/0505041.

[42] R. Vidunas, Askey-Wilson relations and Leonard pairs, Technical Report MHF 200517, Kyushu University 2005; arXiv:math.QA/0511509.

[43] A.S. Zhedanov, "Hidden symmetry" of Askey-Wilson polynomials, Teoret. Mat Fiz. 89 (1991) 190-204.

Kazumasa Nomura

College of Liberal Arts and Sciences

Tokyo Medical and Dental University

Kohnodai, Ichikawa, 272-0827 Japan

email: knomura@pop11.odn.ne.jp

Paul Terwilliger

Department of Mathematics

University of Wisconsin

480 Lincoln Drive

Madison, Wisconsin, 53706 USA

email: terwilli@math.wisc.edu

Keywords. Leonard pair, tridiagonal pair, $q$-Racah polynomial, orthogonal polynomial. 2000 Mathematics Subject Classification. 05E35, 05E30, 33C45, 33D45. 